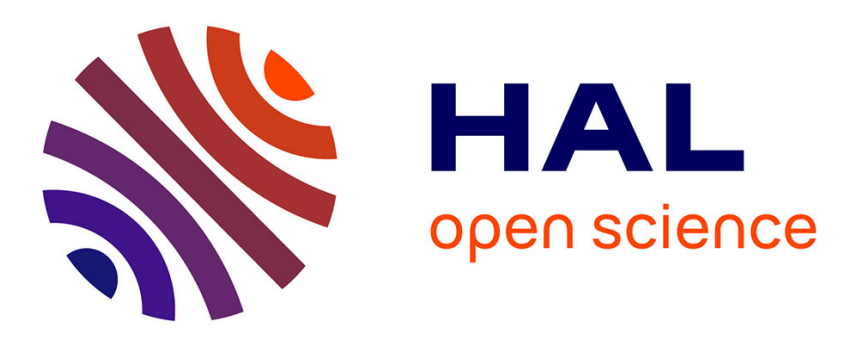

\title{
Numerical study of viscoelastic mixtures through a Cahn-Hilliard flow model
}

Franck Boyer, Laurent Chupin, Pierre Fabrie

\section{To cite this version:}

Franck Boyer, Laurent Chupin, Pierre Fabrie. Numerical study of viscoelastic mixtures through a Cahn-Hilliard flow model. European Journal of Mechanics - B/Fluids, 2004, 23 no 5, pp 759-780. hal-00004087

\section{HAL Id: hal-00004087 https://hal.science/hal-00004087}

Submitted on 27 Jan 2005

HAL is a multi-disciplinary open access archive for the deposit and dissemination of scientific research documents, whether they are published or not. The documents may come from teaching and research institutions in France or abroad, or from public or private research centers.
L'archive ouverte pluridisciplinaire HAL, est destinée au dépôt et à la diffusion de documents scientifiques de niveau recherche, publiés ou non, émanant des établissements d'enseignement et de recherche français ou étrangers, des laboratoires publics ou privés. 


\title{
Numerical study of viscoelastic mixtures through a Cahn-Hilliard flow model
}

\author{
Franck Boyer ${ }^{1}$, Laurent Chupin ${ }^{2}{ }^{3}$ Pierre Fabrie $^{2}$,
}

\begin{abstract}
In this paper we are concerned with the numerical study of viscoelastic diphasic flows.
We first propose a complete model and give some formal justifications including the case where both or one phase is a non-Newtonian one. Then, we have implemented a numerical method taking into account the lagrangian transport in the resolution of the extra-stress equation of Oldroyd type. We describe this fully-practical method and prove its stability so that one can perform long-time numerical simulations.

Several significant tests (channel under shear, filling of tanks, breakup of jets...) illustrate the pertinency of the model and of the numerical method. We show that the results are in accordance with the various qualitative behaviors observed in physical experiments.
\end{abstract}

Key Words - Cahn-Hilliard model, Navier-Stokes equations, Oldroyd model, Numerical approximation.

AMS Subject Classification - 76A10, 76D05, 76M20, 76T10, 81T80.

\section{Introduction}

Following some previous works in this area $[3,6,10,30]$, we propose a physical and mathematical model for the study of diphasic viscoelastic flows. In this model, the sharp interface is replaced by a narrow transition layer across which the fluids may mix. The model describes a flow of a binary mixture, and the behavior of the interface is determined by both diffusion and convection phenomena. The behavior of viscoelastic fluids cannot be predicted by the means of usual Newton's constitutive law since they possess a memory of past deformations which is not the case for Newtonian fluids. Consequently, we have to introduce a more general phenomenological model such as Oldroyd model in addition to the Navier-Stokes equation as a constitutive equation to modelise the viscoelasticity. Let us note that other models could have been used, see [1, 31]. Moreover, as we are dealing with mixtures of two fluids (or two phases), an additional scalar field, the order parameter, is introduced to describe the concentration of one of the fluids in the mixture. The resulting system of equations couples the Navier-Stokes equations, the Oldroyd differential constitutive law and the Cahn-Hilliard equation. In this model both components are supposed to be incompressible but their densities might be differents. In the literature such models are often called quasi-incompressible models (see [23]).

The paper is organised as follows: In section 2, we give a derivation of the Cahn-Hilliard / Navier-Stokes / Oldroyd system. One of the key-point in this derivation process is the adimensionalisation of the equations. It let us find out, depending on the values of four characteristics dimensionless numbers, which terms are really significant in the equations and have to be conserved in the final model. The details of this procedure are given in [3] in the case of Newtonian phases, and so we only give here a brief description.

In section 3, a splitting approach is presented for the numerical computation of the flow. This scheme is primarily an extension of the one proposed in [3] taking into account the Oldroyd equation. We are specifically interested in the discretisation of the Oldroyd differential constitutive law, and we propose an exponential scheme for the time discretisation. This kind of discretisation is supposed to be more representative of the Lagrangian nature of Oldroyd's rheological law. Finally, we prove that the time discretisation we propose is stable under classical conditions. Henceforth, our numerical method can be used to compute large time behavior of the system.

Section 4 is devoted to numerical experiments. A first numerical result is presented for the case of a plane couette flow. This simulation illustrates well-known differences of behavior between a Newtonian fluid and

\footnotetext{
${ }^{1}$ Laboratoire d'Analyse, Topologie et Probabilités, UMR 6632 CNRS/Université de Provence, 39 rue Joliot-Curie, 13453 Marseille Cedex 13, France

${ }^{2}$ Mathématiques Appliquées de Bordeaux, UMR 5466 CNRS/Université Bordeaux 1, 351 cours de la libération, 33405 Talence cedex, France

${ }^{3}$ corresponding author: chupin@math.u-bordeaux.fr
} 
a viscoelactic one. In particular, we observe that the linear velocity profile (which is clearly a stationnary solution of the equations) might be unstable in the viscoelastic case whereas it is unconditionnaly stable in the Newtonian one. We are perfectly in accordance with the results described in [14] and [11].

In the diphasic framework, we first consider the spinodal decomposition under shear. This phenomenon consists in the spontaneous separation of phases which occurs when one quenches rapidly the temperature under the critical temperature of a given mixture. It is well known (and confirmed in numerical simulations in [3]) that when a shear is applied to a mixture under spinodal decomposition, we obtain a structure in parallel layers of each phase. We show the same kind of behavior in the case of the mixture of a viscoelastic fluid with a Newtonian fluid (polymer / solvent for example). We highlight here how the rheological properties of each phase influences the velocity profile in each layer. These results proves in particular that the model and the numerical method proposed are able (for reasonable computational resources) to simulate such situations.

One of the reason of the interest we bring in viscoelastic mixtures flows lies in a tremendous technological importance of drops and bubbles formation in mixing, spraying, and chemical processing, which leads to applications such as ink-jet printing, fiber elongation, and silicon chip technology. We thus present two numerical applications in this perspective.

First of all, we describe and simulate an experiment of fibre stretching, that we compared to experimental results realised by A. Colin and O. Greffier [7]. In this case we are interested in knowing whether the fibre will or will not break, depending on the various parameters of the experiment. We obtain numerically a "phase diagram" very similar to the one obtained in the physical experiments for the Newtonian case. Then we observe that in the viscoelastic case, the corresponding diagram is slightly different and that the fibre may present different kinds of instabilities.

Finally, we consider a simulation of filling of tanks, in which we see that viscoelastic effects enduce a much more important formation of gaz inclusions than in the Newtonian case.

\section{Governing equations}

In this section, we present a system of equations for the study of binary non-Newtonian incompressible flows.

\subsection{Constitutive law for the stress tensor}

We begin with the description of the constituive laws we will use in the sequel to describe the viscoelastic behavior of the fluids. We first consider the case of a single viscoelastic fluid.

Newton's law for the so-called Newtonian fluids, express that the stress tensor applied on a fluid element is essentially proportionnal to the deformation tensor of the fluid element. In more complex fluids, the nonNewtonian ones, we have to prescribe a more general constitutive equation which links stress and deformation. Let us remind here that we only take into account the purely mechanical properties of the fluid we consider. In particular, we suppose that the flow is isothermal so that the effect of temperature in the model does not appear directly. In order to obtain a system of partial differential equations, we will concentrate on the differential forms of these constitutive laws and we will not consider more general integral constitutive laws.

We want to emphasize the fact that there exists in the literature a wide variety of rheological models depending on the fluid we consider but also on the physical situation we study. Each one is an empirical law which has been found to be relevant in the precise situation under study.

\subsubsection{Classical monophasic viscoelastic models}

The classical way to introduce the rheological properties of viscoelastic fluids, is to compare any elementary fluid element to a monodimensional mechanical system composed by springs and dashpots (see [18]). A spring and dahshpot in series is called a Maxwell element (see Figure 1). The spring constant is denoted by $G$ so that the force applied to the spring is $G \gamma_{1}$ where $\gamma_{1}$ is the strain of the spring from its equilibrium state. The force applied to the dashpot is $\eta \partial_{t} \gamma_{2}$ where $\eta$ is the viscosity and $\partial_{t} \gamma_{2}$ is the velocity, that is to say the time 


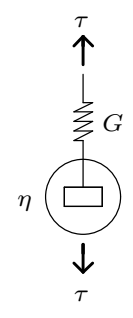

$(1)$

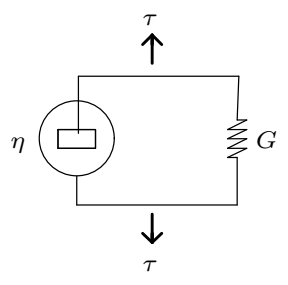

$(2)$

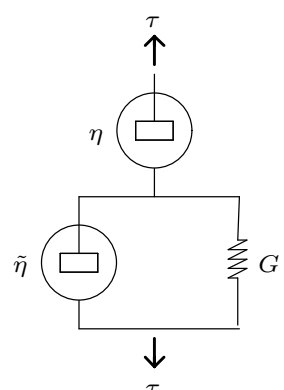

(3)

Figure 1: Maxwell (1), Voigt (2) and Jeffrey (3) elements

rate of change of $\gamma_{2}$, which is the strain of the dashpot. Because they are in series, the two forces are equal and we deduce

$$
\lambda \frac{\partial \tau}{\partial t}+\tau=\eta \frac{\partial \gamma}{\partial t}
$$

where $\lambda=\eta / G$ is a relaxation time, $\gamma=\gamma_{1}+\gamma_{2}$ the total strain and $\tau$ the total stress to which is submitted the system. We can say that this system has instantaneous elasticity in the sense that there is an initial resistance due to the spring.

A Voigt model is a spring and a dashpot in parallel. In this case, the two forces must be added to obtain the total stress, and we have $\gamma_{1}=\gamma_{2}$. We deduce, the following relation

$$
\tau=G \gamma+\eta \frac{\partial \gamma}{\partial t}
$$

where $\gamma=\gamma_{1}=\gamma_{2}$. This element is instantaneously viscous and this model is relevant for viscoelastic solids but not for fluids.

Jeffreys'model is a dashpot and a Voigt model in series and is widely used in the physical models for fluids. It is the model that we shall use in this paper. The total strain $\gamma$ in a Jeffreys'element is the sum of the strain in the first dashpot (of viscosity $\eta$ ) and the one in the Voigt element. Hence, we can write

$$
\gamma=\gamma_{1}+\gamma_{2}
$$

Because these two elements are serial, the forces in both elements are the same:

$$
\tau=\eta \frac{\partial \gamma_{1}}{\partial t}=G \gamma_{2}+\widetilde{\eta} \frac{\partial \gamma_{2}}{\partial t}
$$

After eliminating $\gamma_{1}$ and $\gamma_{2}$, we get an equation connecting the force $\tau$ and the strain $\gamma$ :

$$
\frac{\eta+\widetilde{\eta}}{G} \frac{\partial \tau}{\partial t}+\tau=\eta\left(\frac{\partial \gamma}{\partial t}+\frac{\widetilde{\eta}}{G} \frac{\partial^{2} \gamma}{\partial t^{2}}\right)
$$

We may define a relaxation time as $\lambda=\frac{\eta+\widetilde{\eta}}{G}$ and a retardation time $\widetilde{\lambda}=\frac{\widetilde{\eta}}{G}$.

In order to apply these simple 1D models of mechanical systems to fluid elements, we use the fact that the evolution of the strain $\gamma$ of an elementary fluid element is linked to the velocity field of the fluid as shown in the figure 2 (see [18]). 


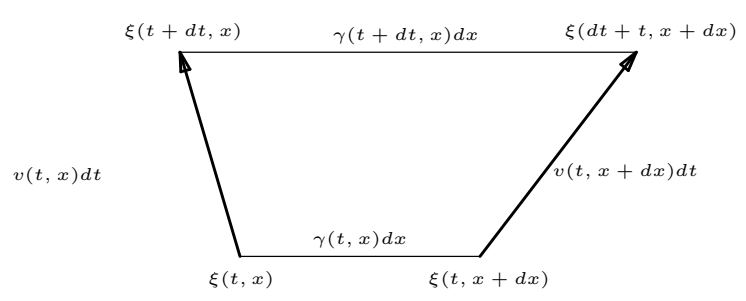

Figure 2: mechanical movement of a cell

Indeed, the strain and the velocity can be defined using the displacement $\xi(x, t)$ giving, at the time $t$, the position of an element which was initially in position $x$. We clearly have the relations

$$
\gamma(t, x)=\frac{\partial \xi}{\partial x}(t, x), \quad v(t, x)=\frac{\partial \xi}{\partial t}(t, x)
$$

and so we deduce that

$$
\frac{\partial \gamma}{\partial t}(t, x)=\frac{\partial v}{\partial x}(t, x)
$$

Finally, the relation (2.1) become

$$
\lambda \frac{\partial \tau}{\partial t}+\tau=\eta\left(\frac{\partial v}{\partial x}+\widetilde{\lambda} \frac{\partial^{2} v}{\partial t \partial x}\right)
$$

As usual, this $1 D$ simplified rheological equation is extended to a physical $3 D$ model in a straightforward way

$$
\lambda \frac{D \boldsymbol{\tau}}{D t}+\boldsymbol{\tau}=2 \eta D(\mathbf{v})+2 \eta \tilde{\lambda} \frac{D}{D t} D(\mathbf{v}) .
$$

Of course, the relation (2.1) is formulated in the lagrangian variables. That is the reason why in (2.2) we have introduced a time derivative $\frac{D}{D t}$ which can be any convective derivative.

In fact, we require that equations should be form invariant under galilean transformation representing a change of frame. A properly invariant tensor rate can be obtained if we interpret the rate operator as the time derivative $\frac{\partial}{\partial t}$ with respect to a reference frame suitably fixed to a body (see [27]). Different choices of body-fixed frames in this interpretation yield different invariant tensor rates and so different models. We present some classical models of the literature (see [18]):

$$
\frac{D \mathbf{M}}{D t}=\frac{\partial \mathbf{M}}{\partial t}+\mathbf{v} \cdot \nabla \mathbf{M}-W(\mathbf{v}) \cdot \mathbf{M}+\mathbf{M} \cdot W(\mathbf{v})-a(D(\mathbf{v}) \cdot \mathbf{M}+\mathbf{M} \cdot D(\mathbf{v})) .
$$

In this formula, $a$ is a rheological parameter varying between -1 and $1, D(\mathbf{v})$ and $W(\mathbf{v})$ stand for the symmetric and skew-symmetric part of $\nabla \mathbf{v}$, respectively. The choices $a=1,-1$, and 0 correspond respectively to the so-called upper-convected derivative, lower-convected derivative, and corotational derivative.

\section{Remark 2.1}

- Since there is no couple forces in our situation, we know that the tensor $\boldsymbol{\tau}$ is symmetric. We can easily notice that the evolution equation for this tensor is invariant by transposition, and so, if $\boldsymbol{\tau}$ is initially symmetric, it will remain symmetric along the time.

- The integral form of this model is known as the Oldroyd model [27].

- It is important to see that the equations describing the evolution of the stress tensor are purely phenomenological. Many other models were developed. Some authors showed that there is an additive term of diffusive type in the Oldroyd model [22, 29]. They derive this term from an analysis of the microscopic dumbell models. Other models like reptation models, or pom-pom polymers model in the case of a high concentration of polymers in a solvant $[20,24,31]$ were derived by using the tube model with a thermodynamical approach. 
- From Jeffrey's model, we can recover the Maxwell model when $\widetilde{\lambda}=0$.

In the case of a Newtonian fluid $(\lambda=\widetilde{\lambda} \approx 0)$, the stress tensor writes: $\boldsymbol{\tau}=2 \eta D(\mathbf{v})$, which is of course the classical Newtonian law. It is classical to split the stress tensor into two parts: the first corresponding to the Newtonian stress, the other one in the extra-stress: $\boldsymbol{\tau}=2 \eta \frac{\widetilde{\lambda}}{\lambda} D(\mathbf{v})+\boldsymbol{\sigma}$. In this formula, the quantity $\eta \frac{\tilde{\lambda}}{\lambda}$ plays the role of an equivalent viscosity. From now on, we introduce a retardation parameter $r=1-\frac{\tilde{\lambda}}{\lambda}$. From (2.2) we deduce

$$
\begin{aligned}
& \boldsymbol{\tau}=2 \eta(1-r) D(\mathbf{v})+\boldsymbol{\sigma} \\
& \frac{D \boldsymbol{\sigma}}{D t}+\frac{\boldsymbol{\sigma}}{\lambda}=\frac{2 \eta r}{\lambda} D(\mathbf{v}) .
\end{aligned}
$$

\section{$2.2 \quad$ Final model}

We have now to take into account the presence of the two phases in the mixture. Among many possibilities, we choose to consider a diffuse interface model. This kind of model have a real thermodynamical origin and let us describe finally the system only with continuous quantities which may have large variations only through the interface. In particular, such models are known to be very well suited to numerical simulations (see for example [3]).

Consider two incompressible fluids, denoted respectively by the subscript 1 and 2 in the following. In order to describe the mixture, we introduce the volumic part of one of the two fluids in the mixture. This quantity, the order parameter, is defined by

$$
\Phi=\Phi(t, x)=\frac{d V_{1}}{d V}
$$

where $d V_{1}$ is the volume filled by the fluid 1 in the elementary volume $d V$. As numerous authors, we shall in fact work with the renormalised order parameter $\varphi=2 \Phi-1$.

We define the mean density and velocity in the mixture and according to the computations performed in [3], the equations of mass conservation for both fluids give two equations: one describing the evolution in time of the order parameter, the other expressing the incompressibility of the mixture.

Then, we write the momentum conservation equation for each phase, by distinguishing the exterior forces and the interior forces. Following the philosophy of diffuse interface models, after having introduce the mean pressure, the mean viscosity... we obtain two equations, the first on the mean velocity $\mathbf{v}$ and the other on the perturbation velocity $\mathbf{w}=\mathbf{v}_{1}-\mathbf{v}_{2}$. We do not give the details of these equations here as one can find the complete computations in [3] and [6]. The key-point is that, once we have obtained these equations with a high number of different terms, we have to perform a dimensional analysis of the system, in order to separate the significant terms and the negligeable terms (for a given range of physical parameters).

In order to get a dimensionless model, we introduce the quantities surmounted by a bar as characteristic quantities supplied by the physical data of the problem ( $\bar{\rho}$ corresponds to the volumic mass of the phase 1 , noted $\rho_{1}^{0}, \bar{v}$ is the shear velocity imposed on the boundary in the case of a couette system, $\bar{L}$ is the size of the domain, $\bar{\xi}$ the caracteristic size for the friction forces between the fluids...). Moreover, it is natural to introduce the characteristic width of the interface $l$ (see [9]) which is typically very small in front of $\bar{L}$. A first dimensionless parameter is then given by the ratio $\alpha=l / \bar{L}$. Furthermore, we deduce the characteristic time of the flow $\bar{T}=\bar{L} / \bar{v}$ from the characteristic length and velocity. We also consider the ratio between the two characteristic velocities $\beta=\bar{w} / \bar{v}, \bar{w}$ being the caracteristic relative velocity between the two phases. Finally, 
we introduce the classical dimensionless numbers defined by (see $[3,6])$ :

$$
\begin{aligned}
& \mathcal{R} e=\bar{\rho} \bar{v} \bar{L} / \bar{\eta}, \text { the Reynolds number, } \\
& \mathcal{K}=\beta \alpha \bar{\xi} \bar{T} / 2 \bar{\rho}^{2} \text {, the capillarity number, } \\
& \mathcal{P} e=\rho_{2}^{0} / \beta \rho_{1}^{0} \text {, the Peclet number, } \\
& \mathcal{W} e=\mathcal{W} e(\varphi)=\lambda(\varphi) / \bar{T} \text {, the Weissenberg number depending on } \varphi .
\end{aligned}
$$

We deduce a system coupling the order parameter $\varphi$, the mean velocity $\mathbf{v}$ and the extra-stress tensor $\boldsymbol{\sigma}$ that we write in the following dimensionless form:

$$
\left\{\begin{array}{l}
\rho(\varphi)=1-\left(\frac{\rho_{2}^{0}-\rho_{1}^{0}}{\bar{\rho}}\right) \frac{1-\varphi}{2} \\
\frac{\partial \varphi}{\partial t}+\mathbf{v} \cdot \nabla \varphi-\frac{1}{\mathcal{P} e} \operatorname{div}\left(\frac{B(\varphi)}{\rho} \nabla\left(\frac{\mu}{\rho}\right)\right)=0 \\
\mu=-\alpha^{2} \Delta \varphi+F^{\prime}(\varphi), \\
\rho\left(\frac{\partial \mathbf{v}}{\partial t}+\mathbf{v} \cdot \nabla \mathbf{v}\right)-\frac{1}{\mathcal{R} e} \operatorname{div}(2 \eta(\varphi)(1-r(\varphi)) D(\mathbf{v}))+\nabla p-\operatorname{div} \boldsymbol{\sigma} \\
\quad=\rho \mathbf{g}+\mathcal{K}\left(\frac{\rho_{2}^{0}-\rho_{1}^{0}}{\bar{\rho}}\right) \frac{1-\varphi^{2}}{2} \nabla\left(\frac{\mu}{\rho}\right)+\mathcal{K} \mu . \nabla \varphi
\end{array}\right.
$$

$$
\operatorname{div}(\mathbf{v})=0,
$$$$
\frac{D \boldsymbol{\sigma}}{D t}+\frac{\boldsymbol{\sigma}}{\mathcal{W} e(\varphi)}=\frac{2 \eta(\varphi) r(\varphi)}{\mathcal{R} e \mathcal{W} e(\varphi)} D(\mathbf{v})
$$

where $B(\varphi)=\left(1-\varphi^{2}\right)^{2} / 8 \xi(\varphi)$ is called mobility coefficient,

and $F(\varphi)=\frac{\varphi^{4}}{4}-\frac{\varphi^{2}}{2}$ is the Cahn-Hilliard potential describing the thermodynamical interactions between the two phases (see [4]).

The structure of this system is now very clear : it consists in the coupling of three evolution equations: a Cahn-Hilliard equation supplemented by a transport term, the Navier-Stokes equations with a capillary term and a viscoelastic stress term and finally an Oldroyd equation giving the evolution of the viscoelastic stress tensor with relaxation/retardation properties.

\subsection{Boundary conditions}

In order to complete the mathematical description of viscoelastic mixtures, we must specify appropriate boundary conditions. The nature of those boundary conditions is intimately connected to the mathematical nature of the governing equations. The physical problem imposes natural conditions on the boundary of the domain for the unknowns $\varphi, \mu$ and $\mathbf{v}$. The no-diffusion through the wall is expressed on the boundary by the relation

$$
\frac{\partial \mu}{\partial n}=0
$$

$n$ being the outward normal to the boundary of the domain. The quantity $\partial_{n} \varphi$ is closely linked to the value of the contact angle of the interface with the boundary. In all the numerical experiments, we choose the boundary condition

$$
\frac{\partial \varphi}{\partial n}=0
$$


which imposes locally the interface to be orthogonal to the boundary. For the velocity, we shall choose whether conditions of Dirichlet type or conditions of Neumann type (see for instance the boundary conditions for an injection test on figure 12 in section 4.3):

$$
\mathbf{v}=\mathbf{v}_{b} \quad \text { or } \quad \frac{\partial \mathbf{v}}{\partial n}=0 \text { in the case of free outflow. }
$$

There is no complete mathematical theory which can guide us for the selection of boundary conditions for the viscoelastic tensor $\boldsymbol{\sigma}[12,19]$. Indeed, the concept of memory fluids clearly suggests that the classical boundary conditions are not sufficient in a viscoelastic flow problem which contains an inlet boundary. The flow inside such a domain is affected by what happened to the fluid upstream.

Nevertheless, in all our computations, the only inlet boundary conditions we may consider are of Poiseuille type. In this case, we can suppose that the fluid upstream lies in an infinite channel and is submitted to a constant pressure gradient. Under this assumption on the past evolution of the upstream flow, we can write the $1 \mathrm{D}$ equations satisfied by the velocity profile $y \mapsto u(y)$ and by the extra-stress tensor $y \mapsto \boldsymbol{\sigma}(y)$. These computations (see [32]) are very similar to the study of section 4.1 with homogeneous boundary conditions in place of shear boundary conditions. In the non-Newtonian case, one can show that the classical velocity profile in a Poiseuille flow simulation $(u(y)=C y(y-1))$ is no more a steady-state solution of these equations. Nevertheless one can impose this profile as an inlet boundary condition for the velocity and the corresponding boundary conditions for the stress tensor $\boldsymbol{\sigma}(y)=\frac{1}{\mathcal{R} e}\left(\begin{array}{cc}\alpha(y) & \beta(y) \\ \beta(y) & \gamma(y)\end{array}\right)$, that is

$$
\left\{\begin{array}{l}
\beta(y)=\frac{r u^{\prime}(y)}{1+k^{2}\left(u^{\prime}(y)\right)^{2}} \\
\alpha(y)=-\mathcal{W} e(1-a) \beta(y) u^{\prime}(y) \\
\gamma(y)=\mathcal{W} e(1+a) \beta(y) u^{\prime}(y)
\end{array}\right.
$$

\section{Numerical scheme}

In this section, the numerical approximation of this system of equations in $2 \mathrm{D}$ is investigated. In all the cases, the computational domain is rectangular but, depending on the physical case we want to describe, we may choose different boundary conditions. From now on, we need to introduce the two components of the velocity $\mathbf{v}=\left(\begin{array}{l}u \\ v\end{array}\right)$

\subsection{Time discretisation}

The time discretisation of the previous system is realised with a fractional step discretisation method. It consists in three main steps.

- Cahn-Hilliard equation: Knowing the values of $\varphi^{n}, \mu^{n}$ and $\mathbf{v}^{n}$ at the instant $t^{n}$, the first step consists in determining the solution of the Cahn-Hilliard equation without the transport term. The method consists essentially in the $\theta$-method with $\theta=0.6$ so as to insure asymptotic stability. A fixed point method is used to solve the non-linear system obtained (see [3]).

For the transport term in this first equation (as for the transport terms in the other equations), we use a scheme of Runge-Kutta type. We are going to describe the method on the example of the transport equation of the order parameter :

$$
\frac{\partial \varphi}{\partial t}+\mathbf{v} \cdot \nabla \varphi=0
$$


The classical Runge-Kutta method used here is a three-stage method of third order. This explicit process can be given by

$$
\varphi^{n+1}=\varphi^{n}-d t K\left(\varphi^{n}\right)+\frac{1}{2} d t^{2} K^{2}\left(\varphi^{n}\right)-\frac{1}{6} d t^{3} K^{3}\left(\varphi^{n}\right),
$$

where $K(f)=\mathbf{v} . \nabla f$. We propose a spatial discretisation of this operator in the section 3.2.2 which ensures the stability of the scheme under a C.F.L. condition.

- Navier-Stokes equations: The non-linear term in the Navier-Stokes equations is treated just like any other convection term in the system as described previously, that is to say

$$
(\mathbf{v} \cdot \nabla \mathbf{v})^{n}=K\left(\mathbf{v}^{n}\right)-\frac{1}{2} d t K^{2}\left(\mathbf{v}^{n}\right)+\frac{1}{6} d t^{2} K^{3}\left(\mathbf{v}^{n}\right) .
$$

For the other terms, the method used is implicit for the pressure and for the viscous terms and explicit for the inertial term and coupling terms as follows:

$$
\left\{\begin{array}{l}
\rho^{n+1}\left(\frac{\mathbf{v}^{n+1}-\mathbf{v}^{n}}{d t}\right)-\frac{1}{\mathcal{R} e} \operatorname{div}\left(2 \eta^{n+1}\left(1-r^{n+1}\right) D\left(\mathbf{v}^{n+1}\right)\right)+\nabla p^{n+1} \\
\quad=\operatorname{div} \boldsymbol{\sigma}^{n}-\rho^{n+1}(\mathbf{v} \cdot \nabla \mathbf{v})^{n}+\rho^{n+1} \mathbf{g}+\frac{\rho_{1}^{0}-\rho_{2}^{0}}{\bar{\rho}} \mathcal{K} \frac{1-\left(\varphi^{n+1}\right)^{2}}{2} \nabla\left(\frac{\mu^{n+1}}{\rho^{n+1}}\right)+\mathcal{K} \mu^{n+1} \cdot \nabla \varphi^{n+1}, \\
\operatorname{div}\left(\mathbf{v}^{n+1}\right)=0,
\end{array}\right.
$$

where we introduced $\rho^{n+1}=\rho\left(\varphi^{n+1}\right), \eta^{n+1}=\eta\left(\varphi^{n+1}\right)$ and $r^{n+1}=r\left(\varphi^{n+1}\right)$. Finally, solving a general Stokes problem is needed and is made by an augmented lagrangian method.

- Oldroyd equation: The last step of this time discretisation consists in determining $\boldsymbol{\sigma}^{n+1}$. The transport term being decoupled and treated as the one in the Cahn-Hilliard equation, it remains to solve the following equation:

$$
\frac{\partial \boldsymbol{\sigma}}{\partial t}-W(\mathbf{v}) \cdot \boldsymbol{\sigma}+\boldsymbol{\sigma} \cdot W(\mathbf{v})-a(D(\mathbf{v}) \cdot \boldsymbol{\sigma}+\boldsymbol{\sigma} \cdot D(\mathbf{v}))+\frac{\boldsymbol{\sigma}}{\mathcal{W} e(\varphi)}=\frac{2 \eta(\varphi) r(\varphi)}{\mathcal{R} e \mathcal{W} e(\varphi)} D(\mathbf{v}),
$$

where $\mathbf{v}, \varphi$ are known at time $t^{n+1}$, and $\boldsymbol{\sigma}$ is known at time $t^{n}$. By making the identification between the set of the two-order tensors and the set of vectors of $\mathbb{R}^{4}$, we can write the previous equation under the following vectorial form:

$$
\frac{\partial \boldsymbol{\sigma}}{\partial t}+\left(\frac{1}{\mathcal{W} e(\varphi)} \mathbf{I} \mathbf{d}-\mathbf{M}_{a}\right) . \boldsymbol{\sigma}=\frac{2 \eta(\varphi) r(\varphi)}{\mathcal{R} e \mathcal{W} e(\varphi)} D(\mathbf{v})
$$

where the matrix $\mathbf{M}_{a}$ is defined by

$$
\mathbf{M}_{a}=\left(\begin{array}{cccc}
2 a \frac{\partial u}{\partial x} & w+a z & w+a z & 0 \\
-w+a z & 0 & 0 & -w-a z \\
-w+a z & 0 & 0 & -w-a z \\
0 & w-a z & w-a z & 2 a \frac{\partial v}{\partial y}
\end{array}\right), \quad w=\frac{1}{2}\left(\frac{\partial v}{\partial x}-\frac{\partial u}{\partial y}\right), \quad z=\frac{1}{2}\left(\frac{\partial v}{\partial x}+\frac{\partial u}{\partial y}\right)
$$

It is easy to determine the solution of the linear equation verified by $\sigma$ :

$$
\begin{aligned}
\boldsymbol{\sigma}^{n+1} & =\boldsymbol{\sigma}\left(t^{n}+d t\right)=e^{-d t\left(\frac{1}{\mathcal{W e}\left(\varphi^{n+1}\right)} \mathbf{I} \mathbf{d}-\mathbf{M}_{a}^{n+1}\right)} \cdot \boldsymbol{\sigma}^{n} \\
& +\int_{0}^{d t} \frac{2 \eta\left(\varphi\left(t^{n}+s\right)\right) r\left(\varphi\left(t^{n}+s\right)\right)}{\mathcal{R} e \mathcal{W} e\left(\varphi\left(t^{n}+s\right)\right)} \exp \left((s-d t)\left(\frac{1}{\mathcal{W} e\left(\varphi\left(t^{n}+s\right)\right)} \mathbf{I d}-\mathbf{M}_{a}\left(t^{n}+s\right)\right)\right) \cdot D\left(\mathbf{v}\left(t^{n}+s\right)\right) d s
\end{aligned}
$$


It is then necessary to approximate the integral in the second member. We choose here to use a method allowing to obtain good results in the case where the Weissenberg number becomes very small, which is the case for Newtonian fluids for example. If we note $\mathbf{I}$ the concerned integral, we used the following approximation

$$
\begin{aligned}
\mathbf{I} & \approx \frac{2 \eta^{n+1} r^{n+1}}{\mathcal{R} e \mathcal{W} e\left(\varphi^{n+1}\right)}\left(\int_{0}^{d t} e^{(s-d t)\left(\frac{1}{\mathcal{W} e\left(\varphi^{n+1}\right)} \mathbf{I d}-\mathbf{M}_{a}^{n+1}\right)} d s\right) \cdot D\left(\mathbf{v}^{n+1}\right) \\
& \approx \frac{2 \eta^{n+1} r^{n+1}}{\mathcal{R} e \mathcal{W} e\left(\varphi^{n+1}\right)}\left(\frac{1}{\mathcal{W} e\left(\varphi^{n+1}\right)} \mathbf{I} \mathbf{d}-\mathbf{M}_{a}^{n+1}\right)^{-1}\left(\mathbf{I} \mathbf{d}-e^{-d t\left(\frac{1}{\mathcal{W} e\left(\varphi^{n+1}\right)} \mathbf{I d}-\mathbf{M}_{a}^{n+1}\right)}\right) \cdot D\left(\mathbf{v}^{n+1}\right) \\
& \approx \frac{2 \eta^{n+1} r^{n+1}}{\operatorname{Re} \mathcal{W} e\left(\varphi^{n+1}\right)} d t e^{-d t\left(\frac{1}{\mathcal{W} e\left(\varphi^{n+1}\right)} \mathbf{I} \mathbf{d}-\mathbf{M}_{a}^{n+1}\right)} \cdot D\left(\mathbf{v}^{n+1}\right) .
\end{aligned}
$$

We obtain the exponential scheme :

$$
\boldsymbol{\sigma}^{n+1}=e^{-d t\left(\frac{1}{\mathcal{W} e\left(\varphi^{n+1}\right)} \mathbf{I d}-\mathbf{M}_{a}^{n+1}\right)} \cdot \boldsymbol{\sigma}^{n}+\frac{2 \eta^{n+1} r^{n+1}}{\mathcal{R} e \mathcal{W} e\left(\varphi^{n+1}\right)} d t e^{-d t\left(\frac{1}{\mathcal{W} e\left(\varphi^{n+1}\right)} \mathbf{I d}-\mathbf{M}_{a}^{n+1}\right)} \cdot D\left(\mathbf{v}^{n+1}\right) .
$$

The next step consists in determining the exponential of the matrix $\mathbf{M}_{a}$. Although the computations are rather long, it is easy to compute its eigenvectors and eigenvalues. We obtain

$$
e^{t \mathbf{M}_{a}}=\left(\begin{array}{cccc}
\alpha^{2} & s \alpha & s \alpha & s^{2} \\
r \alpha & \alpha \beta & r s & s \beta \\
r \alpha & r s & \alpha \beta & s \beta \\
r^{2} & r \beta & r \beta & \beta^{2}
\end{array}\right),
$$

where

$$
\Omega=a^{2}\left(\frac{\partial u}{\partial x}\right)^{2}+a^{2} z^{2}-w^{2}
$$

and

$$
\begin{gathered}
\alpha= \begin{cases}\operatorname{ch}(t \sqrt{\Omega})+a \frac{\partial u}{\partial x} \operatorname{sh}(t \sqrt{\Omega}) / \sqrt{\Omega} & \text { if } \Omega \geq 0, \\
\cos (t \sqrt{-\Omega})+a \frac{\partial u}{\partial x} \sin (t \sqrt{-\Omega}) / \sqrt{\Omega} & \text { if } \Omega \leq 0,\end{cases} \\
\beta= \begin{cases}\operatorname{ch}(t \sqrt{\Omega})-a \frac{\partial u}{\partial x} \operatorname{sh}(t \sqrt{\Omega}) / \sqrt{\Omega} & \text { if } \Omega \geq 0, \\
\cos (t \sqrt{-\Omega})-a \frac{\partial u}{\partial x} \sin (t \sqrt{-\Omega}) / \sqrt{\Omega} & \text { if } \Omega \leq 0,\end{cases} \\
r=(a z-w) \times \begin{cases}\operatorname{sh}(t \sqrt{\Omega}) / \sqrt{\Omega} & \text { if } \Omega \geq 0, \\
\sin (t \sqrt{-\Omega}) / \sqrt{\Omega} & \text { if } \Omega \leq 0,\end{cases} \\
s=(a z+w) \times \begin{cases}\operatorname{sh}(t \sqrt{\Omega}) / \sqrt{\Omega} & \text { if } \Omega \geq 0, \\
\sin (t \sqrt{-\Omega}) / \sqrt{\Omega} & \text { if } \Omega \leq 0 .\end{cases}
\end{gathered}
$$

\section{Remark 3.1}

The discretisation obtained shows that the symmetry property of the system is preserved. More precisely, for the discrete model as for the continuous model, if the initial data $\boldsymbol{\sigma}(0)$ is symmetric then $\boldsymbol{\sigma}(t)$ remains symmetric for all time. Of course, in the numerical simulations we will always take a symmetric initial data.

\subsection{Space discretisation}

\subsubsection{Mesh description and boundary conditions}

Recall that we have supposed that the conditions on the boundary of the domain for the unknowns $\varphi, \mu$ and $\mathbf{v}$ are

$$
\frac{\partial \mu}{\partial n}=0, \quad \frac{\partial \varphi}{\partial n}=0, \quad \mathbf{v}=\mathbf{v}_{b} \quad \text { or } \quad \frac{\partial \mathbf{v}}{\partial n}=0
$$



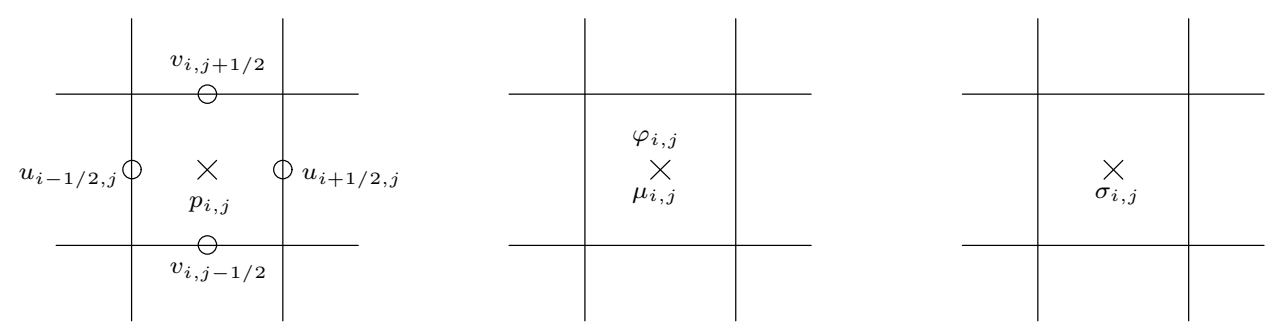

Figure 3: Cell $(i, j)$ and location of the unknows

More precisely, we consider a classical MAC mesh which consists in $N \times M$ uniform cells where the unknowns are taken either in the center of the cell, or in the middle of the edges (see figure 3 ).

In order to adapt easily the method to various boundary conditions, it is useful to introduce artificial unknowns around the physical domain: we introduce for example for $\varphi$ the unknows $\varphi_{0, j}, \varphi_{N+1, j}, \varphi_{i, 0}, \varphi_{i, M+1}$ for $j=1, \ldots M$ and $i=0, \ldots, N+1$.

\subsubsection{Scheme for the convection terms}

For convection terms on $\varphi$ and $\boldsymbol{\sigma}$, the Runge-Kutta's scheme of time discretisation presented first (see section 3.1 ) is now associated to a convenient spatial discretisation. We wish to ensure positiveness of the scheme because it is clear that physical meaningful values of $\varphi$ are the ones in the interval $[-1,1]$.

Let us describe the scheme on a 1D example:

$$
\frac{\partial \varphi}{\partial t}+u \frac{\partial \varphi}{\partial x}=0
$$

The classical Runge-Kutta method used here is a three-stage explicit method of third order:

$$
\varphi^{n+1}=\varphi^{n}-d t K\left(\varphi^{n}\right)+\frac{1}{2} d t^{2} K^{2}\left(\varphi^{n}\right)-\frac{1}{6} d t^{3} K^{3}\left(\varphi^{n}\right)
$$

where $K(f)=u \frac{\partial f}{\partial x}$.

If we write the equation (3.3) as follows

$$
\varphi^{n+1}=\frac{1}{3} \varphi^{n}+\frac{1}{2} L\left(\varphi^{n}\right)+\frac{1}{6} L^{3}\left(\varphi^{n}\right)
$$

where $L=I d-d t K$ then, by convex combinations, it is sufficient to choose a discretisation of $L$ which is positive.

In order to keep a centered stucture, we write a centered discretisation for the operator $L$ in the following form:

$$
\begin{aligned}
(L(f))_{i}^{\mathrm{cen}}=f_{i} & -\frac{\lambda}{2}\left(u_{i+1 / 2}^{+} \Delta f_{i+1 / 2}-u_{i-1 / 2}^{+} \Delta f_{i-1 / 2}\right) \\
& -\frac{\lambda}{2}\left(u_{i+1 / 2}^{-} \Delta f_{i+1 / 2}-u_{i-1 / 2}^{-} \Delta f_{i-1 / 2}\right) \\
& -\lambda\left(u_{i-1 / 2}^{+} \Delta f_{i-1 / 2}-u_{i+1 / 2}^{-} \Delta f_{i+1 / 2}\right)
\end{aligned}
$$

where we have defined $\lambda=d t / \delta x, \Delta f_{i+1 / 2}=f_{i+1}-f_{i}$ and for splitting the diffusive and the antidiffusive terms we have used the notation $u=u^{+}-u^{-}, u^{ \pm} \geq 0$. Following [13], we limit the antidiffusive terms and 
consider the scheme

$$
\begin{aligned}
(L(f))_{i}=f_{i} & -\frac{\lambda}{2}\left(\theta_{i+1 / 2}^{+} u_{i+1 / 2}^{+} \Delta f_{i+1 / 2}-\theta_{i-1 / 2}^{+} u_{i-1 / 2}^{+} \Delta f_{i-1 / 2}\right) \\
& -\frac{\lambda}{2}\left(\theta_{i+1 / 2}^{-} u_{i+1 / 2}^{-} \Delta f_{i+1 / 2}-\theta_{i-1 / 2}^{-} u_{i-1 / 2}^{-} \Delta f_{i-1 / 2}\right) \\
& -\lambda\left(u_{i-1 / 2}^{+} \Delta f_{i-1 / 2}-u_{i+1 / 2}^{-} \Delta f_{i+1 / 2}\right) .
\end{aligned}
$$

We take for the limiters $\theta_{i+1 / 2}^{+}$and $\theta_{i-1 / 2}^{-}$a function of the ratio of two consecutive gradients

$$
\begin{cases}\theta_{i+1 / 2}^{+}=\theta\left(p_{i+1 / 2}\right), & p_{i+1 / 2}=\Delta f_{i-1 / 2} / \Delta f_{i+1 / 2} \\ \theta_{i-1 / 2}^{-}=\theta\left(q_{i-1 / 2}\right), & q_{i-1 / 2}=\Delta f_{i+1 / 2} / \Delta f_{i-1 / 2}\end{cases}
$$

We know that there exists many limiters such that this scheme is positive (see [13]). In all the numerical computations, we used the following limiter

$$
\theta(p)=1-\min \left(|1-p|, \frac{1}{|1-p|}\right)(1-p) .
$$

Using the incremental formulation, it is easy to prove the following result in the two-dimensional case:

\section{Proposition 3.1}

In $2 D$, the scheme defined by (3.4) and (3.5) is positive under the C.F.L. condition

$$
\frac{d t}{\delta x} \max _{i, j}\left(\left|u_{i+1 / 2, j}\right|+\left|u_{i-1 / 2, j}\right|\right)+\frac{d t}{\delta y} \max _{i, j}\left(\left|v_{i, j+1 / 2}\right|+\left|v_{i, j-1 / 2}\right|\right) \leq 1
$$

As a consequence, under the previous condition, we know that for any $1 \leq p \leq+\infty$, we have

$$
\left|\varphi^{n+1}\right|_{p} \leq\left|\varphi^{n}\right|_{p}
$$

where $|\cdot|_{p}$ denotes the classical discrete $L^{p}$ norm.

For the space discretisation of the term $\mathbf{v} . \nabla \mathbf{v}$ we used the same method. Since the discretisation must be determined on the sides of the cells and not in the center (see figure 3), it is necessary to linearly interpolate values of $u$ or $v$. This procedure is very classical in the numerical approximation of Navier-Stokes equations. Of course, we have a similar stability result than the one of proposition 3.1 in this case.

\subsection{Stability properties of the exponential scheme}

We prove in this part that the discretisation which we have introduced for the Oldroyd differential constitutive law, coupled with the Navier-Stokes equations is stable in the monophasic case.

Let us notice that for the continuous model, to our knowledge, no proof justifies the global existence of solutions in the general case. Nevertheless, an interesting case is the case $a=0$ for which we know that there is global existence of a solution for the continuous problem $[6,16]$. We are going to show that it is the same for the discrete model. Indeed, in the case $a=0$, the matrix $\mathbf{M}_{0}$ is skew-symmetric, and so $e^{t \mathbf{M}_{0}}$ is an unitary matrix.

To prove this stability, we assume that the fluid contains only a single phase (by choosing for example $\varphi=1$ ). We suppose that the rheological coefficient $a$ equals zero (it means that we choose the corotationnal time 
derivative for the stress tensor). In that case, we will study the following system obtained in the monophasic case (i.e. letting $\varphi$ being constant in the system):

$$
\left\{\begin{array}{l}
\frac{\partial \mathbf{v}}{\partial t}+\mathbf{v} \cdot \nabla \mathbf{v}-\frac{2(1-r)}{\mathcal{R} e} \operatorname{div}(D(\mathbf{v}))+\nabla p-\operatorname{div}(\boldsymbol{\sigma})=0 \\
\operatorname{div}(\mathbf{v})=0 \\
\frac{\partial \boldsymbol{\sigma}}{\partial t}+\mathbf{v} \cdot \nabla \boldsymbol{\sigma}+\left(\frac{1}{\mathcal{W} e} \mathbf{I d}-\mathbf{M}_{0}\right) \cdot \boldsymbol{\sigma}=\frac{2 r}{\mathcal{R} e \mathcal{W} e} D(\mathbf{v}) .
\end{array}\right.
$$

In the previous section 3.1, we proposed an exponential time discretisation scheme to treat the constitutive law. We will now concentrate on studying the stability of the following semi-discrete scheme:

$$
\left\{\begin{array}{l}
\frac{\mathbf{v}^{n+\frac{1}{2}}-\mathbf{v}^{n}}{d t}=(\mathbf{v} \cdot \nabla \mathbf{v})^{n} \\
\frac{\mathbf{v}^{n+1}-\mathbf{v}^{n+\frac{1}{2}}}{d t}-\frac{2(1-r)}{\mathcal{R} e} \operatorname{div}\left(D\left(\mathbf{v}^{n+1}\right)\right)+\nabla p^{n+1}=\operatorname{div}\left(\boldsymbol{\sigma}^{n}\right) \\
\operatorname{div}\left(\mathbf{v}^{n+1}\right)=0 \\
\mathbf{v}^{n+1}=0 \text { on } \partial \Omega \\
\frac{\boldsymbol{\sigma}^{n+\frac{1}{2}}-\boldsymbol{\sigma}^{n}}{d t}=(\mathbf{v} \cdot \nabla \boldsymbol{\sigma})^{n} \\
\boldsymbol{\sigma}^{n+1}=e^{-d t\left(\frac{1}{\mathcal{W e}}-\mathbf{M}_{0}\right)} \boldsymbol{\sigma}^{n+\frac{1}{2}}+\frac{2 r d t}{\mathcal{R e} \mathcal{W} e} e^{-\frac{d t}{\mathcal{W e}}} e^{d t \mathbf{M}_{0}} D\left(\mathbf{v}^{n+1}\right)
\end{array}\right.
$$

In this system, the symbols $(\mathbf{v} \cdot \nabla \mathbf{v})^{n}$ and $(\mathbf{v} \cdot \nabla \boldsymbol{\sigma})^{n}$ stand for the spatial discretisation of the corresponding terms described in the previous section. We do not explicit the spatial discretisation of all other terms which is very natural.

\section{Proposition 3.2}

The scheme defined by (3.8) is $L^{2}$-stable under the conditions (3.6) and

$$
d t \leq \frac{(1-r) \mathcal{W} e^{2} \mathcal{R} e}{4 r^{2}}
$$

\section{Proof :}

First of all, thanks to condition (3.6), proposition 3.1 shows that we have

$$
\left\{\begin{array}{l}
\left|\mathbf{v}^{n+\frac{1}{2}}\right|_{2} \leq\left|\mathbf{v}^{n}\right|_{2}, \\
\left|\boldsymbol{\sigma}^{n+\frac{1}{2}}\right|_{2} \leq\left|\boldsymbol{\sigma}^{n}\right|_{2} .
\end{array}\right.
$$

We take the inner product in the discrete $L^{2}$ space of the second equation by $\mathbf{v}^{n+1}$ :

$$
\frac{\left|\mathbf{v}^{n+1}\right|_{2}^{2}-\left|\mathbf{v}^{n+\frac{1}{2}}\right|_{2}^{2}}{2 d t}+\frac{\left|\mathbf{v}^{n+1}-\mathbf{v}^{n+\frac{1}{2}}\right|_{2}^{2}}{2 d t}+\frac{2(1-r)}{\mathcal{R} e}\left|D\left(\mathbf{v}^{n+1}\right)\right|_{2}^{2}=-\left(\boldsymbol{\sigma}^{n}, D\left(\mathbf{v}^{n+1}\right)\right) .
$$

Young's inequality gives the following estimation

$$
\left|\left(\boldsymbol{\sigma}^{n}, D\left(\mathbf{v}^{n+1}\right)\right)\right| \leq \frac{1-r}{2 \mathcal{R} e}\left|D\left(\mathbf{v}^{n+1}\right)\right|_{2}^{2}+\frac{\mathcal{R} e}{2(1-r)}\left|\boldsymbol{\sigma}^{n}\right|_{2}^{2}
$$

so that we have

$$
\frac{\left|\mathbf{v}^{n+1}\right|_{2}^{2}-\left|\mathbf{v}^{n+\frac{1}{2}}\right|_{2}^{2}}{2 d t}+\frac{3(1-r)}{2 \mathcal{R} e}\left|D\left(\mathbf{v}^{n+1}\right)\right|_{2}^{2} \leq \frac{\mathcal{R} e}{2(1-r)}\left|\boldsymbol{\sigma}^{n}\right|_{2}^{2}
$$


Let us now consider the equation on $\boldsymbol{\sigma}^{n+1}$ (last equation of (3.8)), substracting $\boldsymbol{\sigma}^{n+\frac{1}{2}}$ and taking the scalar product with $\boldsymbol{\sigma}^{n+1}$ we get the following identity

$$
\begin{aligned}
\frac{\left|\boldsymbol{\sigma}^{n+1}\right|_{2}^{2}-\left|\boldsymbol{\sigma}^{n+\frac{1}{2}}\right|_{2}^{2}}{2 d t}+\frac{\left|\boldsymbol{\sigma}^{n+1}-\boldsymbol{\sigma}^{n+\frac{1}{2}}\right|_{2}^{2}}{2 d t}=\frac{1}{d t} & \int_{\Omega}\left(e^{-d t\left(\frac{1}{\mathcal{W e}}-\mathbf{M}_{0}\right)}-\mathbf{I d}\right) \boldsymbol{\sigma}^{n+\frac{1}{2}}: \boldsymbol{\sigma}^{n+1} \\
& +\int_{\Omega} \frac{2 r}{\mathcal{R} e \mathcal{W} e} e^{-\frac{d t}{\mathcal{W e}}} e^{d t \mathbf{M}_{0}} D\left(\mathbf{v}^{n+1}\right): \boldsymbol{\sigma}^{n+1} .
\end{aligned}
$$

In reality, the integral terms are discrete integral terms on the mesh, that we do not want to explicit since it is not really necessary.

To estimate the first integral, we use the fact that $e^{d t \mathbf{M}_{0}}$ is an unitary matrix:

$$
\begin{aligned}
& \int_{\Omega}\left(e^{-d t\left(\frac{1}{\mathcal{W} e}-\mathbf{M}_{0}\right)}-\mathbf{I d}\right) \boldsymbol{\sigma}^{n+\frac{1}{2}}: \boldsymbol{\sigma}^{n+1}=\int_{\Omega}\left(e^{-d t\left(\frac{1}{\mathcal{W} e}-\mathbf{M}_{0}\right)}-\mathbf{I d}\right) \boldsymbol{\sigma}^{n+\frac{1}{2}}:\left(\boldsymbol{\sigma}^{n+1}-\boldsymbol{\sigma}^{n+\frac{1}{2}}\right) \\
& +\int_{\Omega}\left(e^{-d t\left(\frac{1}{\mathcal{W} e}-\mathbf{M}_{0}\right)}-\mathbf{I d}\right) \boldsymbol{\sigma}^{n+\frac{1}{2}}: \boldsymbol{\sigma}^{n+\frac{1}{2}} \\
& \leq \frac{1}{2}\left|\boldsymbol{\sigma}^{n+1}-\boldsymbol{\sigma}^{n+\frac{1}{2}}\right|_{2}^{2}+\frac{1}{2}\left|\left(e^{-d t\left(\frac{1}{w e}-\mathbf{M}_{0}\right)}-\mathbf{I d}\right) \boldsymbol{\sigma}^{n+\frac{1}{2}}\right|_{2}^{2}+\int_{\Omega}\left(e^{-d t\left(\frac{1}{w e}-\mathbf{M}_{0}\right)}-\mathbf{I d}\right) \boldsymbol{\sigma}^{n+\frac{1}{2}}: \boldsymbol{\sigma}^{n+\frac{1}{2}} \\
& =\frac{1}{2}\left|\boldsymbol{\sigma}^{n+1}-\boldsymbol{\sigma}^{n+\frac{1}{2}}\right|_{2}^{2}+\frac{1}{2}\left|e^{-d t\left(\frac{1}{w e}-\mathbf{M}_{0}\right)} \boldsymbol{\sigma}^{n+\frac{1}{2}}\right|_{2}^{2}-\frac{1}{2}\left|\boldsymbol{\sigma}^{n+\frac{1}{2}}\right|_{2}^{2} \\
& =\frac{1}{2}\left|\boldsymbol{\sigma}^{n+1}-\boldsymbol{\sigma}^{n+\frac{1}{2}}\right|_{2}^{2}-\frac{1-e^{-\frac{2 d t}{W e}}}{2}\left|\boldsymbol{\sigma}^{n+\frac{1}{2}}\right|_{2}^{2} \text {. }
\end{aligned}
$$

Thanks to Young's inequality we have

$$
\frac{2 r}{\mathcal{R} e \mathcal{W} e} e^{-\frac{d t}{W e}}\left|\left(e^{d t \mathbf{M}_{0}} D\left(\mathbf{v}^{n+1}\right), \boldsymbol{\sigma}^{n+1}\right)\right| \leq \frac{1-r}{2 \mathcal{R} e}\left|D\left(\mathbf{v}^{n+1}\right)\right|_{2}^{2}+\frac{2 r^{2}}{(1-r) \mathcal{R} e \mathcal{W} e^{2}}\left|\boldsymbol{\sigma}^{n+1}\right|_{2}^{2},
$$

and then we get

$$
\frac{\left|\boldsymbol{\sigma}^{n+1}\right|_{2}^{2}-\left|\boldsymbol{\sigma}^{n+\frac{1}{2}}\right|_{2}^{2}}{2 d t}+\frac{1-e^{-\frac{2 d t}{\mathcal{W} e}}}{2 d t}\left|\boldsymbol{\sigma}^{n+\frac{1}{2}}\right|_{2}^{2} \leq \frac{1-r}{2 \mathcal{R} e}\left|D\left(\mathbf{v}^{n+1}\right)\right|_{2}^{2}+\frac{2 r^{2}}{(1-r) \mathcal{R} e \mathcal{W} e^{2}}\left|\boldsymbol{\sigma}^{n+1}\right|_{2}^{2} .
$$

Collecting all together (3.11) and (3.12), we obtain the following estimate

$$
\begin{aligned}
\frac{\left|\mathbf{v}^{n+1}\right|_{2}^{2}-\left|\mathbf{v}^{n+\frac{1}{2}}\right|_{2}^{2}}{2 d t} & +\frac{1-r}{\mathcal{R} e}\left|D\left(\mathbf{v}^{n+1}\right)\right|_{2}^{2}+\frac{\left|\boldsymbol{\sigma}^{n+1}\right|_{2}^{2}-\left|\boldsymbol{\sigma}^{n+\frac{1}{2}}\right|_{2}^{2}}{2 d t}+\frac{1-e^{-\frac{2 d t}{\mathcal{W e}}}}{2 d t}\left|\boldsymbol{\sigma}^{n+\frac{1}{2}}\right|_{2}^{2} \\
& \leq \frac{\mathcal{R} e}{2(1-r)}\left|\boldsymbol{\sigma}^{n}\right|_{2}^{2}+\frac{2 r^{2}}{(1-r) \mathcal{R e} \mathcal{W} e^{2}}\left|\boldsymbol{\sigma}^{n+1}\right|_{2}^{2} \\
& \leq \frac{\mathcal{R} e}{2(1-r)}\left|\boldsymbol{\sigma}^{n}\right|_{2}^{2}+\frac{2 r^{2}}{(1-r) \mathcal{R} e \mathcal{W} e^{2}}\left(\left|\boldsymbol{\sigma}^{n+1}\right|_{2}^{2}-\left|\boldsymbol{\sigma}^{n+\frac{1}{2}}\right|_{2}^{2}\right)+\frac{2 r^{2}}{(1-r) \mathcal{R e \mathcal { W } e ^ { 2 }}}\left|\boldsymbol{\sigma}^{n+\frac{1}{2}}\right|_{2}^{2} .
\end{aligned}
$$

If we assume (3.9), or equivalently

$$
\frac{2 r^{2}}{(1-r) \mathcal{R} e \mathcal{W} e^{2}} \leq \frac{1}{4 d t}
$$

then we deduce

$$
\frac{\left|\mathbf{v}^{n+1}\right|_{2}^{2}-\left|\mathbf{v}^{n+\frac{1}{2}}\right|_{2}^{2}}{2 d t}+\frac{\left|\boldsymbol{\sigma}^{n+1}\right|_{2}^{2}-\left|\boldsymbol{\sigma}^{n+\frac{1}{2}}\right|_{2}^{2}}{4 d t} \leq \frac{\mathcal{R} e}{2(1-r)}\left|\boldsymbol{\sigma}^{n}\right|_{2}^{2}+\frac{2 r^{2}}{(1-r) \mathcal{R} e \mathcal{W} e^{2}}\left|\boldsymbol{\sigma}^{n+\frac{1}{2}}\right|_{2}^{2} .
$$

Indeed, if $\left|\boldsymbol{\sigma}^{n+1}\right|_{2} \geq\left|\boldsymbol{\sigma}^{n+\frac{1}{2}}\right|_{2}$ this result is clear from (3.13) and (3.14) and if $\left|\boldsymbol{\sigma}^{n+1}\right|_{2}<\left|\boldsymbol{\sigma}^{n+\frac{1}{2}}\right|_{2}$, we only have to add to (3.11) the term $\frac{\left|\boldsymbol{\sigma}^{n+1}\right|_{2}^{2}-\left|\boldsymbol{\sigma}^{n+\frac{1}{2}}\right|_{2}^{2}}{4 d t}$ which is negative. 
Now, we use (3.10), to obtain

$$
\frac{\left|\mathbf{v}^{n+1}\right|_{2}^{2}-\left|\mathbf{v}^{n}\right|_{2}^{2}}{2 d t}+\frac{\left|\boldsymbol{\sigma}^{n+1}\right|_{2}^{2}-\left|\boldsymbol{\sigma}^{n}\right|_{2}^{2}}{4 d t} \leq C\left|\boldsymbol{\sigma}^{n}\right|_{2}^{2}
$$

Hence, using the classical discrete Gronwall lemma, we obtain a bound on $\left|\mathbf{v}^{n}\right|_{2}$ and on $\left|\boldsymbol{\sigma}^{n}\right|_{2}$ which does not depend on the time step.

\section{Remark 3.2}

- With the usual parameters $r=0.9, \mathcal{W} e=10$ and $\mathcal{R} e=10$, the second condition of (3.9) writes $d t \lesssim 30$ which is not very forcing.

- Moreover, for Newtonian flows, this condition disappears. Remind that the retardation parameter $r$ is defined as follows:

$$
r=1-\frac{\widetilde{\mathcal{W} e}}{\mathcal{W} e}
$$

where $\widetilde{\mathcal{W} e}$ is the retardation time (We being the relaxation time). We say that the flow is Newtonian (see [18]) when $\widetilde{\mathcal{W} e}=\mathcal{W} e \rightarrow 0$, so that in this situation we have

$$
\frac{(1-r) \mathcal{R} e \mathcal{W} e^{2}}{4 r^{2}}=+\infty
$$

\section{Numerical simulations}

In this final section, we present the results obtained with the model and the scheme previously introduced. Our objective is to show that this model and this numerical method is able to give relevant numerical results. For this reason we compare the results for Newtonian and viscoelastic cases in three kinds of physical situations in which the viscoelastic properties of the fluids are known to be very influent.

\subsection{Plane couette flow: one fluid situation}

Viscoelastic materials have mechanical properties that are intermediate between those of elastic solids and viscous liquids. In this paragraph, we present an exemple of behavior which is not accounted by the theory of Newtonian fluids. The results obtained are presented simultaneously, for comparison purposes, for a Newtonian flow and a viscoelastic flow.

Consider the case of a plane couette flow in the monophasic context. We impose the shear rate and so we consider a periodic channel with shear boundary conditions. The theory of Newtonian flows show that asymptotically, the solution converges to the stationnary solution which consists in a linear velocity profile depending only on the transversal variables.

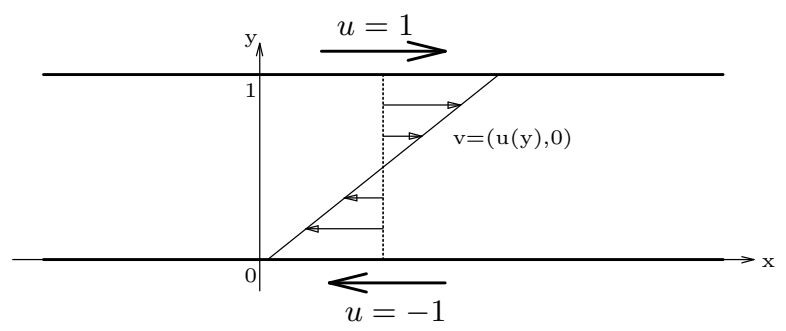

Figure 4: Boundary condition for the velocity in a Couette plane cell 
For a viscoelastic fluid, there is a range of parameters for which the asymptotic behavior is very different. Exactly, there exists, in a certain range of the velocity of the moving plates, an acountable infinity of steady-state solutions in wich the velocity is piecewise linear, the shear stress is constant and the other stress components are characterised by jump discontinuities. We demonstrate here this phenomenon of shear-banding (see for instance $[11,14,28]$ and references therein). More precisely, let us consider the stationary solutions of the equations (with $\varphi \equiv 1$ ), namely the solutions of

$$
\left\{\begin{array}{l}
(\mathbf{v} \cdot \nabla \mathbf{v})-\frac{2(1-r)}{\mathcal{R} e} \operatorname{div}(D(\mathbf{v}))+\nabla p=\operatorname{div} \boldsymbol{\sigma} \\
\operatorname{div} \mathbf{v}=0, \\
\mathcal{W} e(\mathbf{v} \cdot \nabla \boldsymbol{\sigma}-W(\mathbf{v}) \cdot \boldsymbol{\sigma}+\boldsymbol{\sigma} \cdot W(\mathbf{v})-a(D(\mathbf{v}) \cdot \boldsymbol{\sigma}+\boldsymbol{\sigma} \cdot D(\mathbf{v})))+\boldsymbol{\sigma}=\frac{2 r}{\mathcal{R} e} D(\mathbf{v}),
\end{array}\right.
$$

which corresponds to one viscoelastic fluid obeying a Jeffrey constitutive law in a stationary state. Let us recall that the rheological parameter $a$ lies in $[-1,1]$ and the retardation parameter $r$ in $] 0,1[$. The case $r=0$ corresponds to a Newtonian fluid (and in this case $\boldsymbol{\sigma}=0$ ), and the case $r=1$ to a Maxwell model. The following computations are mainly given in [17].

The geometry of a Couette flow is assumed to be invariant under $x$ translations (see figure 4). Invariance under $x$ translations lead us to find a solution depending only on the variable $y$. Incompressibility gives the form $(u(y), 0)$ for the velocity field so that we can write

$$
\mathbf{v}=\left(\begin{array}{c}
u(y) \\
0
\end{array}\right), \quad \boldsymbol{\sigma}=\frac{1}{\mathcal{R} e}\left(\begin{array}{ll}
\alpha(y) & \beta(y) \\
\beta(y) & \gamma(y)
\end{array}\right) .
$$

We easily deduce from (4.1) the equations

$$
\begin{gathered}
-(1-r) u^{\prime}=\beta-C_{0}, \\
\mathcal{R} e p^{\prime}=\gamma^{\prime}, \\
\mathcal{W} e(1-a) \beta u^{\prime}+\alpha=0, \\
\mathcal{W} e((1-a) \gamma-(1+a) \alpha) u^{\prime}+2 \beta=2 r u^{\prime}, \\
-\mathcal{W} e(1+a) \beta u^{\prime}+\gamma=0 .
\end{gathered}
$$

The first two equations must be interpreted in fact as the continuity of the functions $\beta+(1-r) u^{\prime}$ and $\mathcal{R} e p-\gamma$ through the discontinuies of $u^{\prime}$. Moreover, it is interesting to note that in this case the velocity field is independent of the Reynolds number.

This system holds for $y \in] 0,1[$ and is supplemented with the boundary conditions $u(0)=-1$ and $u(1)=1$. These conditions show that the constant $C_{0}$ reads (after integration of (4.2a)):

$$
C_{0}=\int_{0}^{1} \beta(y) d y+2(1-r) .
$$

From (4.2c) and (4.2e), we deduce $\alpha$ and $\gamma$ as functions of $\beta$ and $u^{\prime}$. Using (4.2a) we express $\beta$ as a function of $u^{\prime}$.

$$
\beta=\frac{r u^{\prime}}{1+\mathcal{W} e^{2}\left(1-a^{2}\right)\left(u^{\prime}\right)^{2}}, \quad \alpha=-\mathcal{W} e(1-a) \beta u^{\prime}, \quad \text { and } \quad \gamma=\mathcal{W} e(1+a) \beta u^{\prime} .
$$

We introduce now $Y=k u^{\prime}(y)$ and finally using (4.2d) we readily obtain the following equation for $Y$, where $k^{2}=\mathcal{W} e^{2}\left(1-a^{2}\right)$ and $D=k C_{0}$ (see $[17,21]$ for similar computations):

$$
-(1-r) Y^{3}+D Y^{2}-Y+D=0 .
$$

This equation shows that $u^{\prime}$ can only take at most three values depending on the values of the parameters. Moreover, if we know $u^{\prime}$, from (4.4), the stress-tensor $\boldsymbol{\sigma}$ is completely determined. 
From (4.5), we see that, if $u^{\prime}$ (and so $Y$ ) is known, we have

$$
D=Y\left(1+(1-r) Y^{2}\right) /\left(1+Y^{2}\right)
$$

If we plot the function $f_{r}: Y \mapsto D$, we can see that the equation (4.5) admits three distinct real roots if and only if $r \geq 8 / 9$ and $D^{+}<D<D^{-}, D^{ \pm}$being given by (see figure 5 ):

$$
D^{ \pm}=f_{r}\left(Y^{ \pm}\right), \quad \text { where } \quad\left(Y^{ \pm}\right)^{2}=\frac{3 r-2 \pm \sqrt{r(9 r-8)}}{2(1-r)}, Y^{ \pm} \geq 0 .
$$

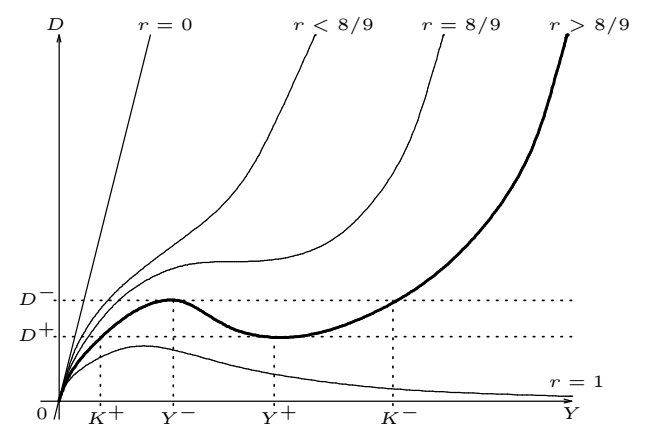

Figure 5: graph of $f_{r}: Y \mapsto D$ for different values of the parameter $r$

On the figure 6 , we represent the viscosity $D / Y$ according to the rate of shearing. The decrease of this function shows that in all the cases, the fluid is a shear-thinning fluid.

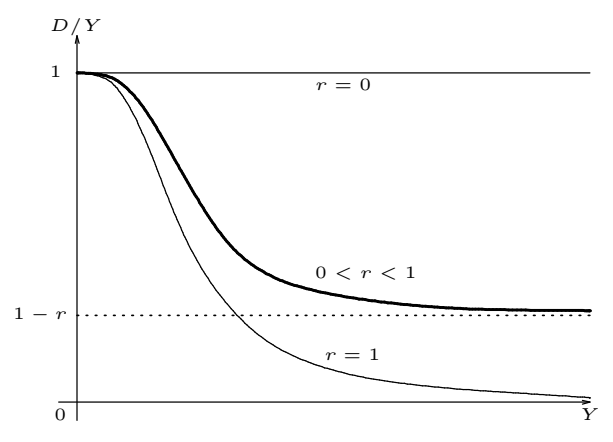

Figure 6: graph of $Y \mapsto D / Y$

- When $r<\frac{8}{9}$, the solution $Y$ of (4.5) is unique, then the two boundary conditions on $u$ show that necessarily, $u^{\prime}(y) \equiv 2$, that is to say $u(y)=2 y-1$. Hence, we have $Y=2 k$ and $D$ is perfectly determined by formula (4.6).

In this case, the situation is similar to the Newtonian one.

- In the case where $r>\frac{8}{9}$, we have seen than there may be up to three values for $Y$. We can show that there exists numerous piecewise linear solutions of the problem.

Let us choose the constant $D$ between $D^{-}$and $D^{+}$, we know that there exists 3 solutions to (4.5) denoted by $Y_{1}, Y_{2}$ and $Y_{3}$, with $Y_{1} \leq Y_{2} \leq Y_{3}$. 
Now we build a velocity profile from these three values of $Y$ (each of them giving a value $Y_{i} / k$ for $u^{\prime}$ ). Consider first a piecewise linear function $u$ defined by

$$
\left\{\begin{array}{l}
u(0)=-1, \\
u^{\prime}(y)=\frac{Y_{1}}{k}, y \in\left[0, \mu_{1}\right], \quad u^{\prime}(y)=\frac{Y_{2}}{k}, y \in\left[\mu_{1}, \mu_{1}+\mu_{2}\right], u^{\prime}(y)=\frac{Y_{3}}{k}, y \in\left[\mu_{1}+\mu_{2}, \mu_{1}+\mu_{2}+\mu_{3}\right] .
\end{array}\right.
$$

Such a function is a stationnary state for the problem if the $\left(\mu_{i}\right)$ are positive and if they satisfy

$$
\left\{\begin{array}{l}
\mu_{1}+\mu_{2}+\mu_{3}=1 \\
\mu_{1} Y_{1}+\mu_{2} Y_{2}+\mu_{3} Y_{3}=2 k \\
\mu_{1} Z_{1}+\mu_{2} Z_{2}+\mu_{3} Z_{3}=D-2 k(1-r)
\end{array}\right.
$$

where $Z_{i}=r Y_{i} /\left(1+Y_{i}^{2}\right)$. Indeed, the first equation ensures that $u$ is defined on $[0,1]$, the second one stands for the boundary condition $u(1)=1$ and the last one is equivalent to (4.3). Our aim is now to find positive solutions to this system.

Since for the polynom (4.5) the sum of the roots is equal to their product, we see that the determinant of the system (4.8) is zero. Furthermore, as we have supposed that the three roots are distinct, it is clear that the rank of the system is 2 . Then, we notice that the second member is always in the range of the system. Indeed, if we impose $\mu_{1}=0$, we find the solution

$$
\mu_{1}=0, \quad \mu_{2}=\frac{Y_{3}-2 k}{Y_{3}-Y_{2}}, \quad \mu_{3}=\frac{2 k-Y_{2}}{Y_{3}-Y_{2}} .
$$

If we impose $\mu_{2}=0$, we find

$$
\mu_{1}=\frac{Y_{3}-2 k}{Y_{3}-Y_{1}}, \quad \mu_{2}=0, \quad \mu_{3}=\frac{2 k-Y_{1}}{Y_{3}-Y_{1}} .
$$

These two particular solutions affinely span the whole set of solutions. As we have supposed $Y_{1} \leq Y_{2} \leq Y_{3}$, it is easily seen that there exists positive solutions to this system if and only if

$$
Y_{1} \leq 2 k \leq Y_{3} .
$$

Remember now, that $D$ is also unknown in this problem and that $Y_{1}, Y_{2}$ and $Y_{3}$ depend on $D$. In conclusion, the parameter $r>\frac{8}{9}$ being fixed, we see on figure 5 , that there exists $D \in \mathbb{R}$ and some non-trivial (that is to say $\mu_{i} \neq 1$ ) positive solutions of (4.8), if and only if

$$
K^{+}<2 k<K^{-}, \quad \text { where } \quad K^{ \pm}=\frac{D^{ \pm}}{(1-r)\left(Y^{ \pm}\right)^{2}} .
$$

For such values of the parameters, we obtain numerous stationnary solutions for the problem whose velocity profile is not linear in contrast with the Newtonian case.

In fact, we can build an infinity of stationnary solutions. Indeed, consider $D$ and $\left(\mu_{1}, \mu_{2}, \mu_{3}\right)$ a non-trivial positive solution of the above system. Let $\omega_{i}$ be an union of intervals of total measure $\mu_{i}$ such that

$$
[0,1]=\omega_{1} \cup \omega_{2} \cup \omega_{3} .
$$

Then we can define a function $u$ with

$$
u(0)=-1, u^{\prime}(y)=\mu_{i} / k \text { if } y \in \omega_{i},
$$

which is another piecewise linear stationnary solution of the problem.

The fact that it may exist a very large number a solutions to the stationnary problem is closed linked to the differences in the large time behavior of the original evolution problem. 
We show now some numerical examples illustrating this more complex asymptotic behavior of viscoelastic fluids under shear in a plane couette cell. Then, we will show the influence of viscoelasticity in a diphasic situation: the spinodal decomposition.

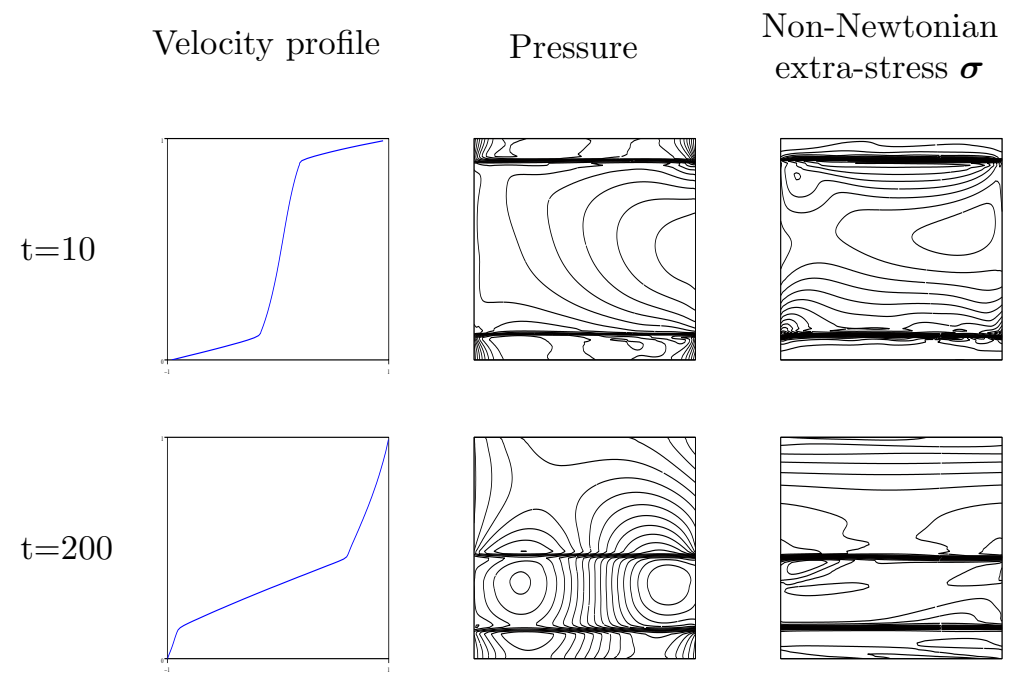

Figure 7: One viscoelastic fluid under shear

The first test realised corresponds to the case of one viscoelastic flow whose parameters precisely lie in the domain of non-uniqueness of stationary solutions $\left(r>8 / 9\right.$ and $\left.K^{+}<2 k<K^{-}\right)$:

$$
r=0.9, \quad \mathcal{W} e=1, \quad a=0 \quad(\text { i.e. } k=1)
$$

All the numerical results presented in this paragraph are obtained with the following parameters: $\mathcal{R} e=100$, $\mathcal{K}=0.01$ and $\mathcal{P} e=1000$. We consider a fluid initially at rest. We show the velocity profile, the pressure and the value of the component $\alpha$ at times $t=10$ and $t=200$ (see figure 7). Furthermore we present the evolution in time of the velocity profile (see figure 8).

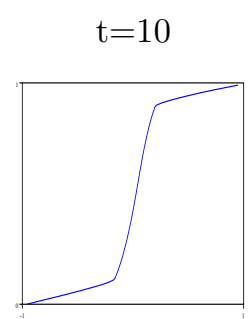

$\mathrm{t}=250$

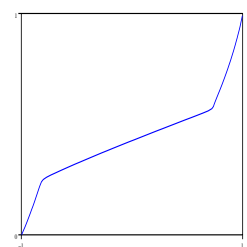

$\mathrm{t}=50$

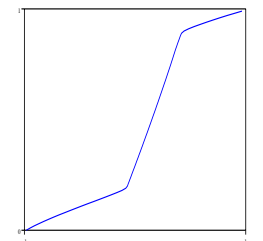

$\mathrm{t}=300$

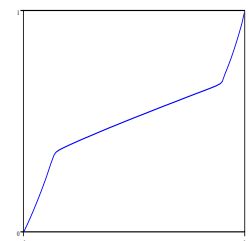

$\mathrm{t}=100$

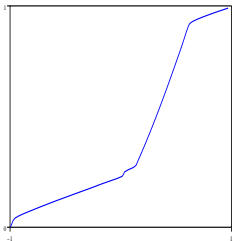

$\mathrm{t}=350$

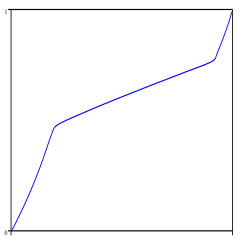

$\mathrm{t}=150$

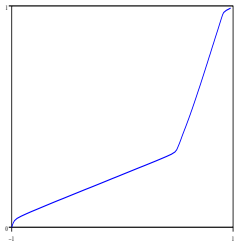

$\mathrm{t}=400$

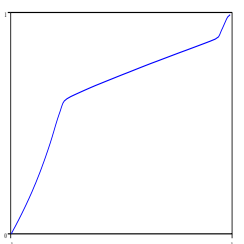

$\mathrm{t}=200$

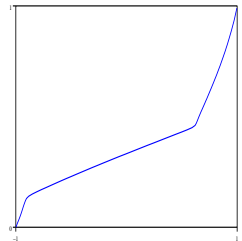

$\mathrm{t}=450$

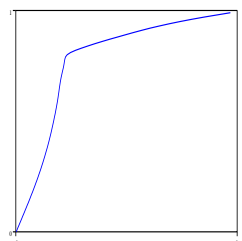

Figure 8: Time evolution of the velocity profile in a one fluid viscoelastic plane couette flow 
We clearly see on figure 8 that the velocity profile does not converge to a stationnary state like in the classical Newtonian case. Nevertheless the structure of this velocity profile is exactly the one obtained in the stationnary analysis above: the solution is essentially piecewise linear with two different slopes which are expected to be the roots of a cubic polynomial function as seen before. One can observe at time $t=100 \mathrm{a}$ slightly more complex profile composed by six linear parts whereas all other results show only three linear parts. Let us emphasize that even in this case, we only have two different slopes of the profile. Such turbulences results can be interpreted as a competition between the viscous effects and the elastic effects (see [15, 26, 33]). In [15], the authors observe experimentally that the flow of a sufficiently elastic polymer solution can become irregular even at low Reynolds number.

Before passing to diphasic simulations, we wish to illustrate numerically the instability of a linear velocity profile. We refer to the paper of C. Guillopé and J.C. Saut (see [17]), in which it is proved a linear stability criterion for the classical linear profile, as well as a nonlinear Lyapounov stability result for the simplified 1D system. We recall here the linear stability analysis.

\section{Proposition 4.1}

For the linear profile solution $\left(u^{\prime}(y) \equiv 2\right)$, the stress tensor $\boldsymbol{\sigma}$ is independent of $y$ and we have

$$
\beta(y)=\beta_{0}=\frac{2 r}{1+4 k^{2}}, \quad \alpha(y)=\alpha_{0}=-2 \mathcal{W} e(1-a) \beta_{0}, \text { and } \gamma(y)=\gamma_{0}=2 \mathcal{W} e(1+a) \beta_{0} .
$$

- If $0 \leq r<8 / 9$, then the solution $\left(u(y)=2 y-1, \alpha_{0}, \beta_{0}, \gamma_{0}\right)$ is linearly stable.

- If $8 / 9 \leq r<1$, then $\left(u(y)=2 y-1, \alpha_{0}, \beta_{0}, \gamma_{0}\right)$ is linearly stable if and only if $0 \leq k<Y_{-}$or $k>Y_{+}$(see (4.7) and figure 5).

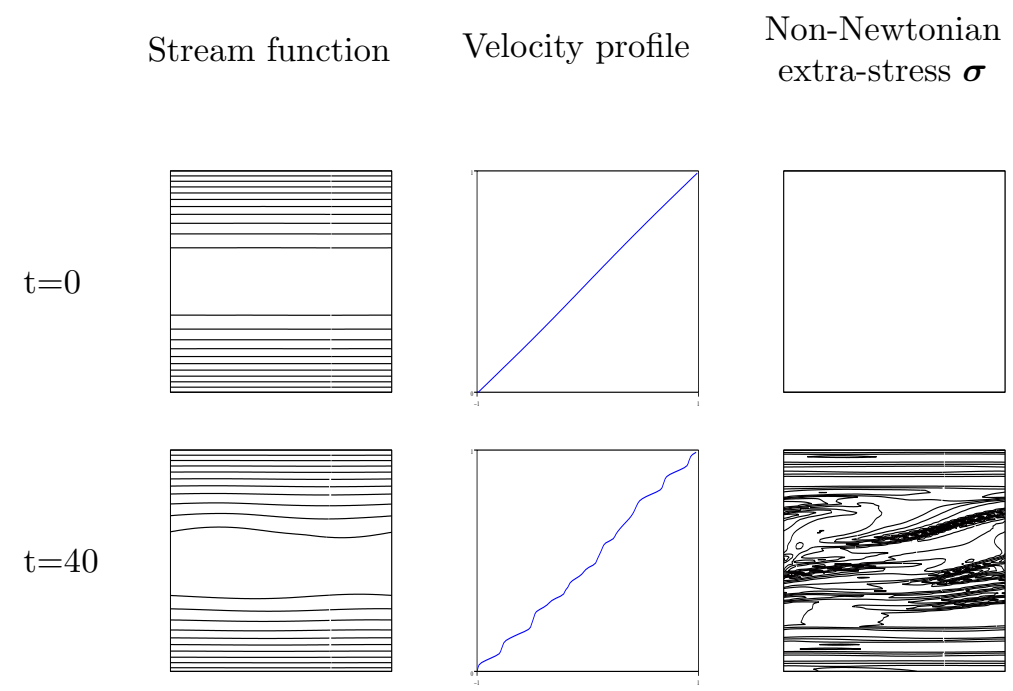

Figure 9: Instability of the linear velocity profile for a viscoelastic fluid under shear

We take an initial data $\left(u(y)=2 y-1, \alpha_{0}, \beta_{0}, \gamma_{0}\right)$ for the $2 \mathrm{D}$ evolution problem and we let it evolve. The rheological parameters $r=0.9, \mathcal{W} e=1$ and $a=0$ ensure that this solution is linearly unstable. This fact is confirmed here, as seen in figure 9 , at time $t=40$.

We observe a destabilized velocity profile which is piecewise linear with only three different slopes but a large number of discontinuities in $u^{\prime}(y)$. As we have seen above, in the stationnary case the possible slopes for the velocity profile can be obtained as solutions of the system (4.8). As the evolution is quite slow in this process, one can expect than the stationnary analytic study of the system (4.8) is not far from being relevant in this evolution problem. 


\subsection{Plane couette flow: spinodal decomposition under shear of viscoelastic phases}

We can enter now into the study of the spinodal decomposition of a two phases mixture under shear. We recall that two phases, above the critical temperature, are miscible but when the temperature is suddenly quenched under the critical temperature then the two phases separate spontaneously. This phenomenon is known as the spinodal decomposition. Without any other exterior influence on the mixture, it is well known that the spinodal decomposition is a two-stages evolution process: a separation stage then a pattern formation stage where the bulk phases organise themselves into macroscopic patterns evolving slowly with many changes of topology.

From some years $[3,8,25]$, it is known that, when one consider a spinodal decomposition process under shear in a couette cell, then the pattern formation is very influenced by the shear and the patterns organise themselves into parallel layers in the shear direction. Furthermore, it is experimentally and numerically proved that the characteristic size of the layers depend on the shear rate: the higher the shear rate is, the thiner the layers are. Previous numerical results have been obtained in [3] in the Newtonian case to illustrate these properties.

As we have seen above, the viscoelasticity of the phases (or one of the phases) may influate a lot the behavior of shear flows in the one fluid case, and so it is natural to think that such influence may also appear clearly in the diphasic case.

Let us show some numerical illustrations of this phenomenon. The initial condition is randomly selected around $\varphi=0$ which is not a metastable state for the Cahn-Hilliard potential $F(\varphi)=\frac{1}{4} \varphi^{4}-\frac{1}{2} \varphi^{2}$ we have chosen. The fact that we consider an under-critical temperature is contained in the double-well structure of the potential. For comparison purposes, this random initial data is the same for all the results presented here. The mixture is initially at rest, that is to say that at time $t=0$, the temperature is quenched to an under-critical value and the shear is started simultaneously.

We present here the result obtained for a mixture of a Newtonian fluid and a viscoelastic fluid. In this case, we remark that the caracteristic time for the apparition of parallel layers is longer than in the purely Newtonian case. In figure 10, we show the results at time $t=10$ and $t=100$. The rheological parameters used for this test are the following

$$
r=0.9, \mathcal{W} e=10, a=0 .
$$

Viscoelastic mixture
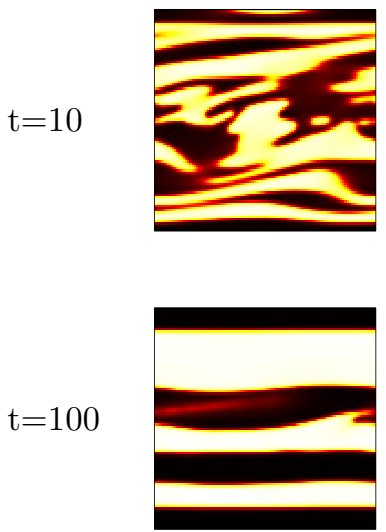
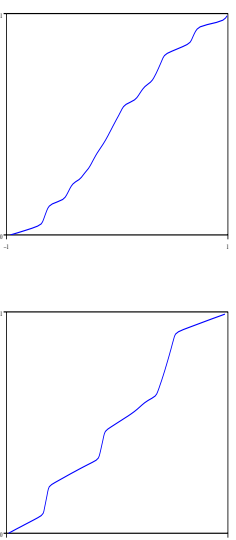

Newtonian mixture
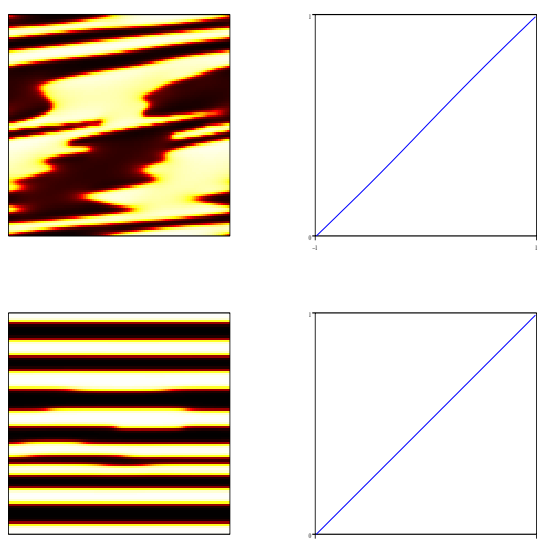

Figure 10: Spinodal decomposition under shear (mixture composition and velocity profile)

On the left hand side of figure 10 we show the order parameter $\varphi$ in gray scale. The white fluid is the Newtonian fluid whereas the black fluid is the viscoelastic fluid. 
If one performs similar analytic computations than in the above one fluid case, one can expect that the velocity profile at large time is piecewise linear with a finite number of possible slopes obtained from the solutions of a cubic polynomial. Such computations are detailed in a sharp interface context in [21].

In this particular example, it seems that a unique given slope is associated to each phase, that is to say that the discontinuities of $u^{\prime}$ are located exactly on the interfaces between the two phases. This phenomenon is quite surprising as we may expect to find more than one slope for the velocity profile in each pure phase layer. Nevertheless, it is not known yet if the analytic computations performed in the sharp interface model can be extended without modifications to the diffuse interface model we use here.

\subsection{Breakup of jets}

The breakup of liquid jets is important in many applications, such as ink-jet printing for example (see [10]). Here we consider the dynamics of an injection of a fluid into another fluid. The experimenter pushes the fluid toward the tip with a given velocity, and at the same time he takes along the nozzle behind with an other given velocity (see figure 11). A liquid jet emanating from this nozzle may break up into droplets if the two velocities are large enough. In the other case, the injection produces a long filament.

This experiment takes place essentially in an horizontal plane (because the depth of the container is small in front of its other caracteristic sizes) so that we can perform 2D numerical simulations without gravity.

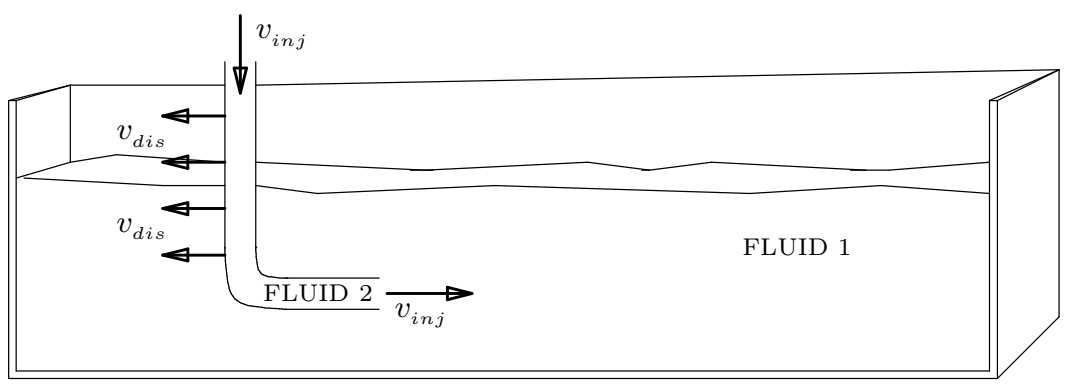

Figure 11: Physical experiment

The computational domain is supposed to be fixed to the nozzle referential (which is deplaced with constant velocity). Furthermore, we have choosen boundary conditions on the velocity as shown in figure 12. Les us emphasize that we impose Neumann boundary conditions at the artificial boundary of the computational domain which stands for free outflow condition.

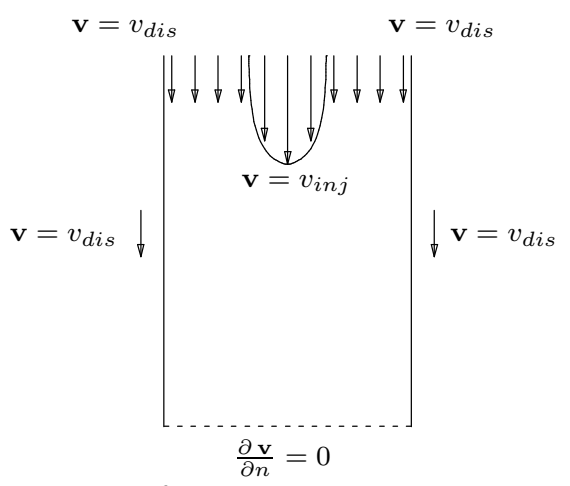

Figure 12: Boundary conditions for the velocity in the breakup of jets case

We introduce two caracteristic velocities of the flow: the injection velocity of the injected fluid and the displacement velocity of the nozzle (that is to say the velocity in the nozzle referential of the fluid initially in the container). In this situation, it is clear that viscosities have a profound influence, not only on the final 
length of the neck, but also on the radii of drops. In the physical experiments [7, 10], we know that the injected fluid is typically 10 times more viscous than the other fluid. We have taken this viscosity ratio in the numerical simulations we present here.

At $t=0$, fluid 2 begins to enter into fluid 1 and the nozzle is displaced to the velocity $v_{\text {dis }}$.

First of all, let us compare the resulting jets with the same physical parameters in the Newtonian and viscoelastic case. For this experiment, we choose $v_{d i s}=v_{i n j}=1$, the other parameters being:

$$
\begin{aligned}
& \mathcal{R} e=100, \mathcal{K}=0.001, \mathcal{P} e=10 \text { and } \\
& \mathcal{W} e=1, r=0.95, a=0, \quad \text { for the non-Newtonian fluid. }
\end{aligned}
$$

$$
\mathrm{t}=1
$$

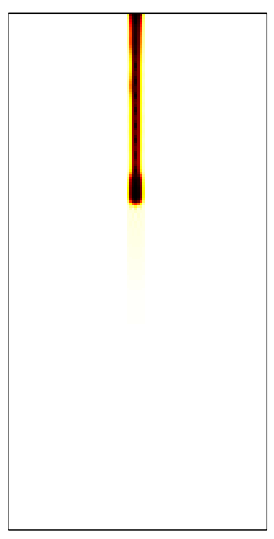

$\mathrm{t}=1.4$

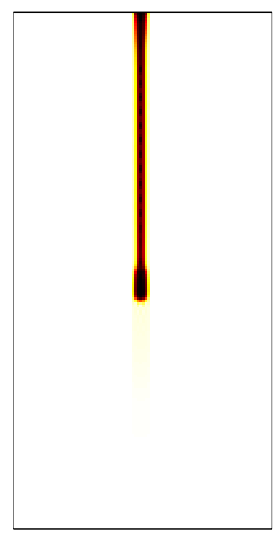

$\mathrm{t}=1.8$

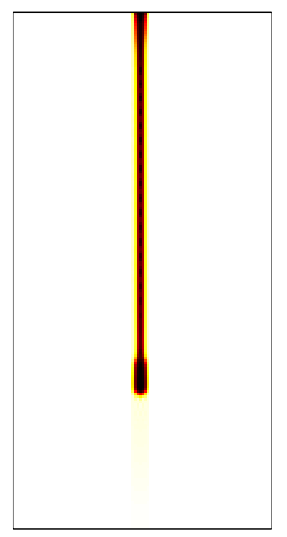

$\mathrm{t}=2.2$

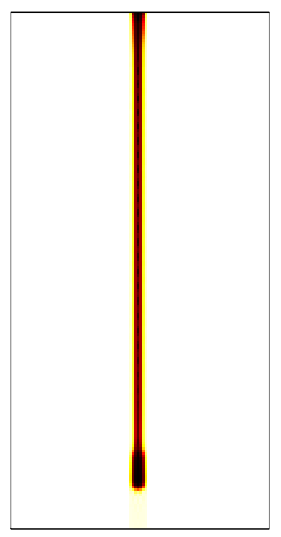

$\mathrm{t}=2.5$

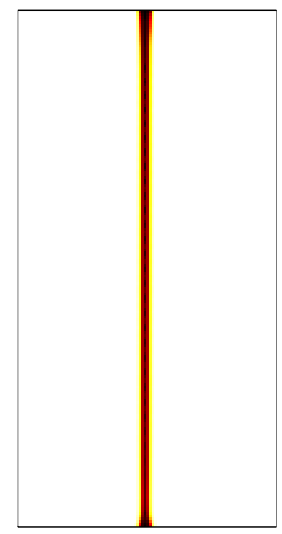

Figure 13: Behavior of the jet for $v_{\text {inj }}=v_{\text {dis }}=1$ : Newtonian case

$\mathrm{t}=1$

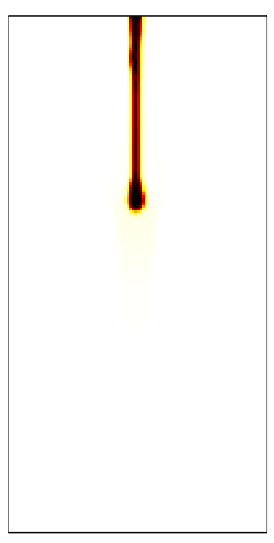

$\mathrm{t}=1.4$

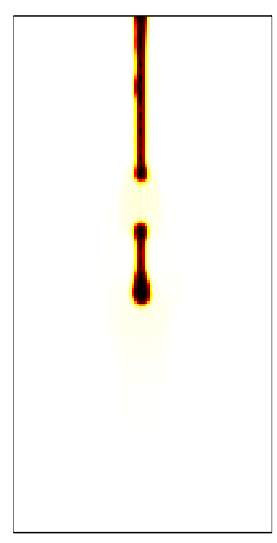

$\mathrm{t}=1.8$

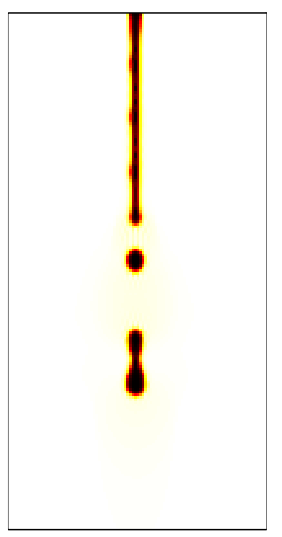

$\mathrm{t}=2.2$

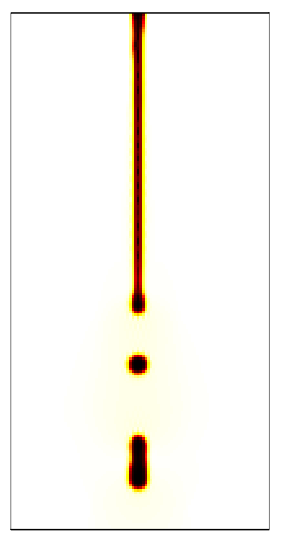

$\mathrm{t}=2.5$

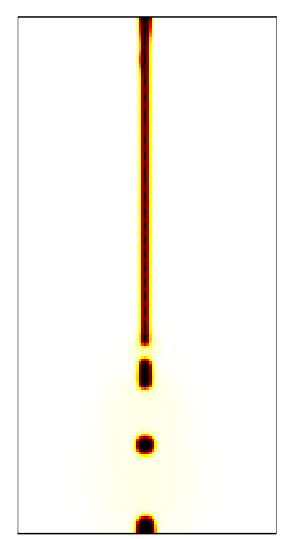

Figure 14: Behavior of the jet for $v_{\text {inj }}=v_{\text {dis }}=1$ : Viscoelastic case

We clearly see that, for these values of the parameters, the Newtonian jet is stable whereas the viscoelastic one breaks up into different parts. In fact these two situations can occur in the Newtonian and in the viscoelastic 
cases. We can observe (experimentally ans numerically) these two different modes: one called continuous mode in which the jet does not break, and the other one called dotted-lines in which the jet breaks up and separate into droplets.

In the purely Newtonian case, the results can be summarised in a phase diagram (left-hand side part of figure 15). This diagram corresponds exactly to the one obtained by the physicists in their experiments [7]. We can see, in the $\left(v_{d i s}, v_{i n j}\right)$-plane, a straight line separating the range of parameters for which we observe the continuous mode and the one for which we observe a dotted-line mode (break up of the jet).

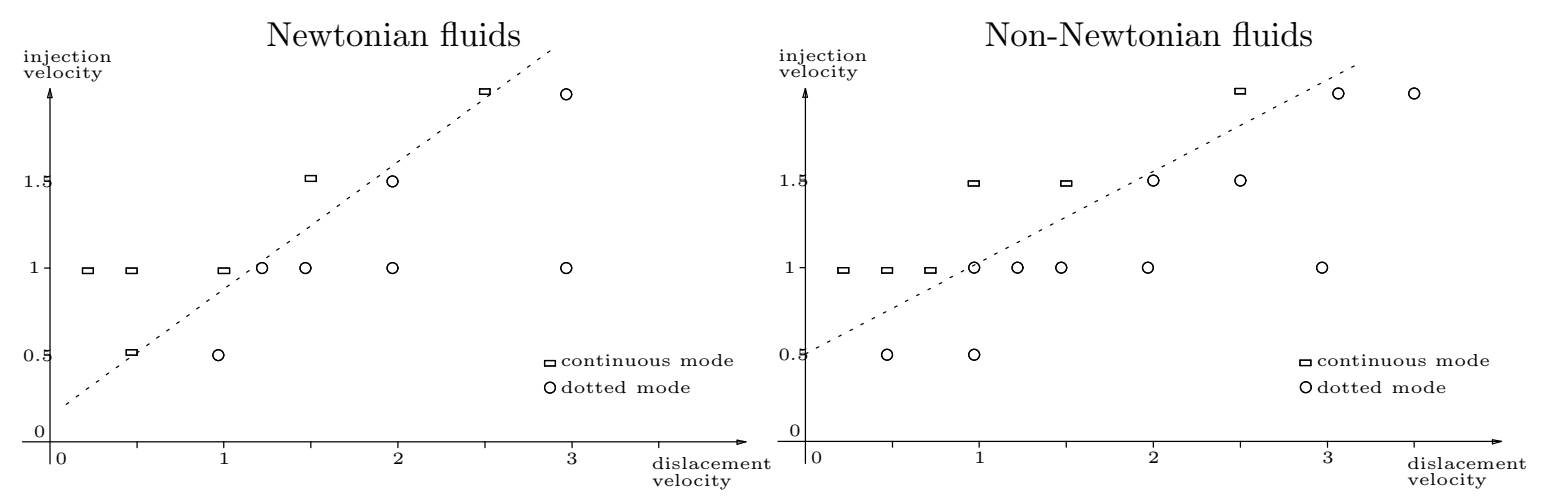

Figure 15: Phase diagrams

Our goal is to compare those results with the visocelastic situation and more precisely when one injects a viscoelastic fluid into a Newtonian fluid. Many differences can be highlighted (see right-hand side diagram in figure 15). First of all, the slope of the line separating the continuous mode part and the dotted line part of the phase diagram is significantly lower in the non-Newtonian case (slope near 0.8 for Newtonian fluid, and near 0.5 for non-Newtonian one). This means that in the viscoelastic case, the jet is more stable than in the Newtonian case in the sense that, to observe a break up of the jet for a given injection velocity, one needs to impose a greater displacement velocity on the nozzle than in the Newtonian case.

$$
\mathrm{t}=0.5 \stackrel{\text { Newtonian fluids }}{\mathrm{t}=2} \quad \begin{gathered}
\mathrm{t}=0.5 \\
\mathrm{t}=2
\end{gathered}
$$
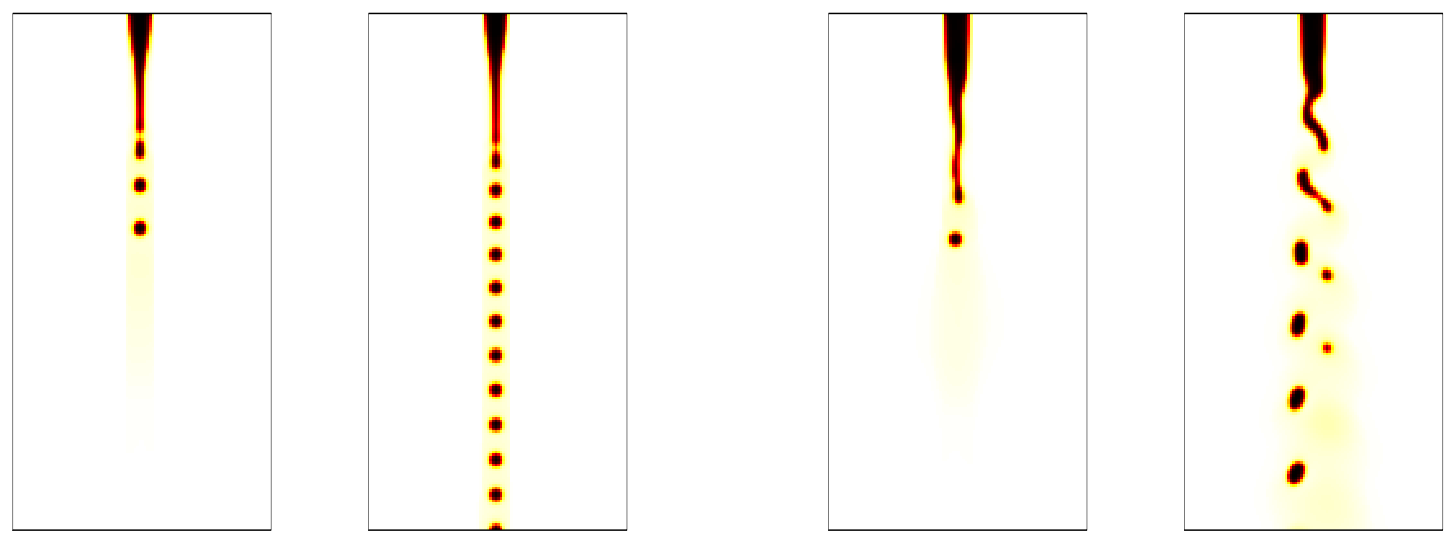

Figure 16: Behavior of a larger jet for $v_{\text {inj }}=1$ and $v_{\text {dis }}=4.5$

Furthermore, if we increase the diameter of the jet, we can observe an other kind of viscoelastic effects. Indeed, in this case, we see undulations growing (Rayleigh instability) on the non-Newtonian jet, as shown on 
figure 16 whereas the Newtonian flow is very regular, for a relatively "small" velocity for a viscoelastic flow injected in a Newtonian flow (the same results are observed in the Newtonian case but only for high velocity). We also observe that the size of the droplets is greater than in the Newtonian case.

\subsection{Filling of a tank}

One of the reasons of the interest we bring in viscoelastic flows lies in a tremendous technological importance of drop formation in mixing, spraying, and chemical processing, which leads to applications such as ink-jet printing, fiber spinnig, and silicon chip technology.

In this last part, the fluid 2 is injected into a tank initially containing the fluid 1 . In all the following simulations, we consider the filling of the tank until the three fifth, then we stop the injection to observe the stabilisation process. Furthermore, the injected fluid has a greater density than the other one and we take into account the gravity in these numerical tests.

In the first result, the two fluids are Newtonian and the injection velocity is $v_{\text {inj }}=3$ (consequently we stop the filling at time $t=6$ ). The physical parameters are given by

$$
\mathcal{R} e=1000, \mathcal{K}=0.0001, \mathcal{P} e=1000, \eta_{1}=10, \eta_{2}=1, \rho_{1}=1000, \rho_{2}=1 .
$$

The result is shown on figure 17 at times $t=3, t=6, t=7$ and $t=9$. We compare then, all the other

$$
\mathrm{t}=3
$$

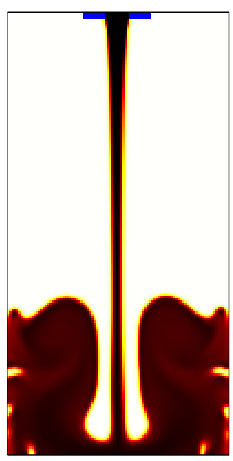

$\mathrm{t}=6$

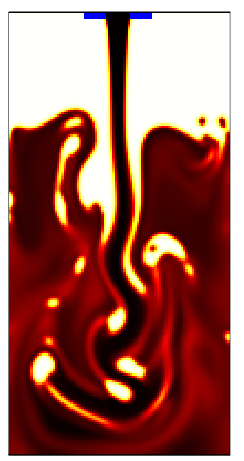

$\mathrm{t}=7$

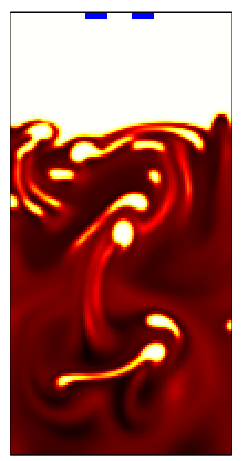

$\mathrm{t}=9$

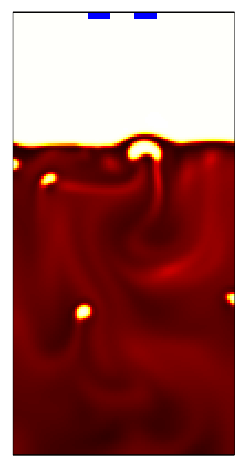

Figure 17: Filling of a tank with a Newtonian fluid

parameters being identical, this result with the case when the two fluids are viscoelastic with rheological parameters:

$$
r=0.95, \mathcal{W} e=1, a=0 .
$$

This non-Newtonian result is shown on figure 18 at the same times than the Newtonian one. The viscoelastic result is very different from the Newtonian one in particular in the first part ( $t=3$ and $t=6)$ of the process in which one can see a much more important quantity of bubbles created in the mixture viscoelastic mixture. This feature of viscoelastic fluids is relatively well-known by physicists and engineers and is the cause of numerous problems in the filling of fuel tank as in some circumstances we can observe some bubbles which remain blocked at the bottom of the tank. 


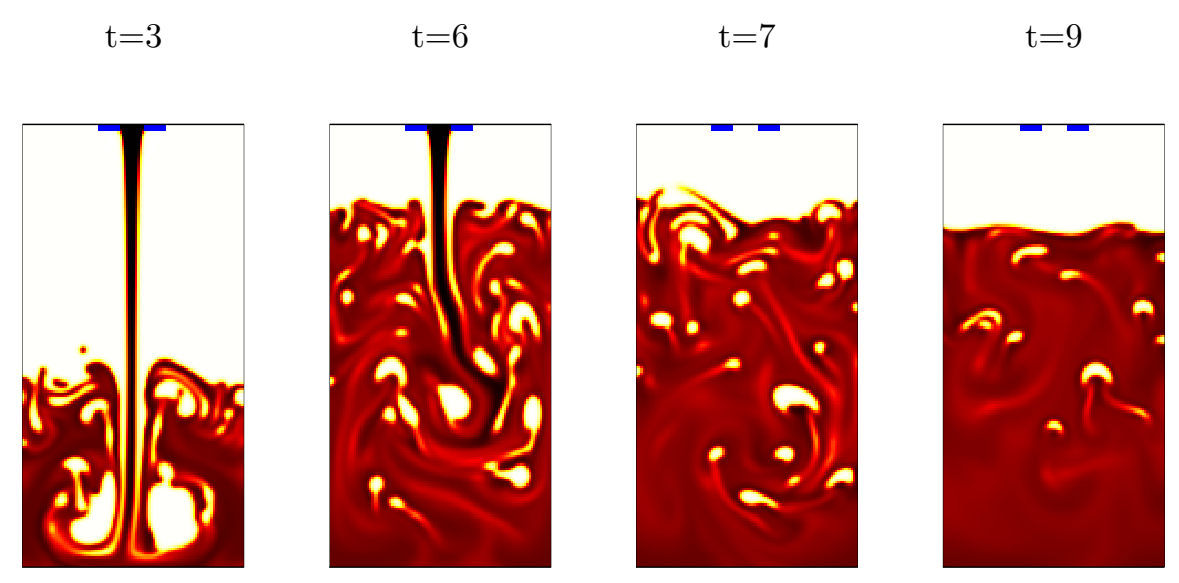

Figure 18: Filling of a tank with visco-elatstic fluid

\section{Conclusions}

In this paper we have proposed a complete mathematical model for the study of mixtures of possibly viscoelastic fluids. This model consists in a diffuse interface diphasic model of Cahn-Hilliard type coupled with a Navier-Stokes equation and a non-Newtonian constitutive law of Oldroyd type. This model is derived from the classical conservation equations for each phase assorted with phenomenological constitutive relations.

In a second part we propose a stable numerical method to perform simulations for the previous model. The Oldroyd differential constitutive law is treated with an exponential scheme that we consider to be more relevant for this rheological equation derived from very simple mechanical systems of spring and dashpots. As far as the spatial discretisation is concerned we choose a high order and positive scheme for the transport part of the equations in order to ensure that the numerical diffusion is limited in long time computations.

In a last part we propose the results of numerical simulations for numerous physical situations in which viscoelasticity is known to play an important role. We deal with the case of spinodal decomposition of a binary non-Newtonian mixture under shear in a plane couette system. Then, we consider the problem of the break up of jets which arises particularly in fibre stretching applications. We show that the model and the numerical scheme are able to retrieve some qualitative experimental results. Finally, we give illustrations of the wide variety of situations that one can consider with this model by performing simulations of tank filling in which it is well known that viscoelastic properties of some kind of fuels for example has a tremendous importance in industrial applications.

Acknowledgment: We want to thank Dr. A. Colin and her team of the Centre de Recherche Paul Pascal (Bordeaux), for many helpful discusions and for having shown us the main results of their experimentations. We also thank the referees for the care they brought to the reading of this paper.

\section{References}

[1] G.B. Bishko, O.G. Harlen, T.C.B. McLeish, T.M. Nicholson, Numerical simulation of the transient flow of branched polymer melts through a planar contraction using the 'pom-pom' model, J. Non-Newtonian Fluid Mech. 82, pp 255-273 (1999)

[2] F. Boyer, Mathematical study of multiphase flow under shear through order parameter formulation, Asymptotic Analysis, Vol. 20 n.2, pp 175-212 (1999) 
[3] F. Boyer, A theoretical and numerical model for the study of incompressible mixture flows, Computers and Fluids Vol. 31 no 1 (2002)

[4] J.W. Cahn, J.E. Hilliard, Free energy of non-uniform system. I. Interfacial free energy, J. Chem. Phys. 28, pp 258-267 (1958)

[5] R. Chella, J. Vinals, Mixing of two-phase fluids by a cavity flow, Physical Review E 53, pp 3832-3840 (1996)

[6] L. Chupin, Existence result for a mixture of non Newtonian flows with stress diffusion using the CahnHilliard formulation, Discrete and Continuous Dynamical Systems B, vol. 3, 1, pp 45-68 (2003)

[7] A. Colin, O. Greffier and S. Manneville, Centre de Recherche Paul Pascal, Université Bordeaux 1, Personal communications

[8] G. Cristobal Azkarate, Etude de quelques changements structuraux dans les fluides complexes à l'équilibre et hors équilibre, Ph. D Thesis, CPMOH, Université Bordeaux 1 (2000)

[9] M. Doi, Dynamics of domains and textures, Theoretical Challenges in the dynamics of complex fluids, pp 293-314 (1997)

[10] J. Eggers, Nonlinear dynamics and breakup of free-surface flows, Rev. of Mod. Physics, Vol.60, No.3, pp 865-929 (1997)

[11] S.M. Fielding, P.D. Olmsted, Flow phase diagrams for concentration-coupled shear banding, Eur. Phys. J. E 11, pp 65-83 (2003)

[12] A. Fortin, M. Fortin, A new approach for the FEM simulation of viscoelastic flows, J. non-Newtonian Fluid Mech., 32, pp 295-310 (1989)

[13] E. Godlewski, P.A. Raviart, Hyperbolic systems of conservation laws, Marketing Editions (1991)

[14] G. C. Georgiou, D. Vlassopoulos, On the stability of the simple shear flow of a Johnson-Segalman fluid, J. Non-Newtonian Fluid Mech., 75, pp 77-79 (1998)

[15] A. Groisman, V. Steinberg, Nature, Vol. 405, pp 53-55 (2000)

[16] C. Guillopé, J.C. Saut, Existence Results for the Flow of viscoelastic Fluids with a differential, Nonlinear Analysis, Vol. 15 n.9, pp 849-869 (1990)

[17] C. Guillopé, J.C. Saut, Global existence and one-dimensional nonlinear stability of shearing motions of viscoelastic fluids of Oldroyd type, Rairo model Math. Anal. Num., Vol. 24 n.3, pp 369-401 (1990)

[18] D. D. Joseph, Fluid Dynamics of Viscoelastic Liquids, Springer (1990)

[19] R. Keunings, Computer Modeling for Polymer Processing, C.L.Tucker III, Hanser Publishers (1989)

[20] R.G. Larson, T.C.B. McLeish, J. Rheol., 42, pp 81-110 (1998)

[21] H. Le Meur, Non-uniqueness and linear stability of the one-dimensional flow of multiple viscoelastic fluids, Math. Model. and Num. Anal., Vol. 31, 2, pp 185-212 (1997)

[22] C.Y.D. Lu, P.D. Olmsted et R.C Ball, Effects of Non-local Stress on the determination of Shear Banding Flow, Physical Review Letters 84, pp. 642-645 (2000)

[23] J.S. Lowengrub, L. Truskinovsky, Quasi-incompressible Cahn-Hilliard fluids and topological transitions, Proc. Royal Soc. of London, Serie A, vol. 454, pp 2617-2654 (1998) 
[24] G. Marrucci, Dynamics of entanglements: A nonlinear model consistent with the Cox-Merz rule, J. nonNewtonian Fluid Mech., 62, pp 279-289 (1996)

[25] K. Maatsuzaka, T. Koga, T. Hashimoto, Phys. Rev. Lett., 80, p 5441 (1998)

[26] S.J.Muller, R.G Larson, E.S.G Shaqfed, Rheol. Acta. 28, pp 499 (1989)

[27] J.G. Oldroyd, On the formation of rheological equations of state, Proc. R. Soc. Lond. A200, pp 523-541 (1950)

[28] P.D. Olmsted, Two-state shear diagrams for complex fluids in shear flow, Europhys. Lett., 48, 3, pp 339-345 (1999)

[29] P.D. Olmsted, C.Y. David Lu, Phase separation of Rigid-Rod Suspensions in Shear Flow, to appear (1999)

[30] A. Onuki, Phase transitions of fluids in shear flow, J. Phys. Condens. Matter 9, pp 6119-6157 (1997)

[31] H.C. Öttinger, Capitalizing on nonequilibrium thermodynamics: branched polymers, XIIIth International Congress on Rheology, Cambridge, UK (2000)

[32] P. Saramito, Simulation numérique d'écoulements de fluides viscoélastiques par éléments finis incompressibles et une méthode de directions alternées-applications, Thesis, I.N.P.Grenoble (1990)

[33] E.S.G. Shaqfed, Annu. Rev. Fluid. Mech. 28 pp 129 (1996) 